\title{
Unveiling the remarkable photodissociation region of Messier $\mathbf{8}^{\star}$
}

\author{
M. Tiwari ${ }^{1, \star \star}$, K. M. Menten ${ }^{1}$, F. Wyrowski ${ }^{1}$, J. P. Pérez-Beaupuits ${ }^{1,2}$, H. Wiesemeyer ${ }^{1}$, \\ R. Güsten ${ }^{1}$, B. Klein ${ }^{1}$, and C. Henkel ${ }^{1,3}$
${ }^{1}$ Max-Planck-Institut für Radioastronomie, Auf dem Hügel, 53121 Bonn, Germany e-mail: mtiwari@mpifr-bonn.mpg.de
2 European Southern Observatory, Alonso de Córdova 3107, Vitacura Casilla 7630355, Santiago, Chile \\ 3 Astronomy Department, King Abdulaziz University, PO Box 80203, Jeddah 21589, Saudi Arabia
}

Received 8 December 2017 / Accepted 25 March 2018

\begin{abstract}
Aims. Messier 8 (M8) is one of the brightest HII regions in the sky. We collected an extensive dataset comprising multiple submillimeter spectral lines from neutral and ionized carbon and from CO. Based on this dataset, we aim to understand the morphology of M8 and that of its associated photodissociation region (PDR) and to carry out a quantitative analysis of the physical conditions of these regions such as kinetic temperatures and volume densities.

Methods. We used the Stratospheric Observatory For Infrared Astronomy (SOFIA), the Atacama Pathfinder Experiment (APEX) $12 \mathrm{~m}$, and the Institut de Radioastronomie Millimétrique (IRAM) $30 \mathrm{~m}$ telescopes to perform a comprehensive imaging survey of the emission from the fine structure lines of $[\mathrm{C} \mathrm{II}]$ and $[\mathrm{C} \mathrm{I}]$ and multiple rotational transitions of carbon monoxide (CO) isotopologs within $1.3 \times 1.3 \mathrm{pc}$ around the dominant Herschel 36 (Her 36) system, which is composed of at least three massive stars. To further explore the morphology of the region, we compared archival infrared, optical, and radio images of the nebula with our newly obtained fine structure line and CO data, and in particular with the velocity information these data provide. We performed a quantitative analysis, using both LTE and non-LTE methods to determine the abundances of some of the observed species, kinetic temperatures, and volume densities.

Results. Bright $\mathrm{CO},[\mathrm{C} \mathrm{II}]$ and $\left[\mathrm{C}_{\mathrm{I}}\right]$ emission have been found toward the HII region and the PDR in M8. Our analysis places the bulk of the molecular material in the background of the nebulosity illuminated by the bright stellar systems Her 36 and 9 Sagitarii. Since the emission from all observed atomic and molecular tracers peaks at or close to the position of Her 36, we conclude that the star is still physically close to its natal dense cloud core and heats it. A veil of warm gas moves away from Her 36 toward the Sun and its associated dust contributes to the foreground extinction in the region. One of the most prominent star forming regions in M8, the Hourglass Nebula, is particularly bright due to cracks in this veil close to Her 36 . We obtain $\mathrm{H}_{2}$ densities ranging from $\sim 10^{4}-10^{6} \mathrm{~cm}^{-3}$ and kinetic temperatures of $100-150 \mathrm{~K}$ in the bright PDR caused by Her 36 using radiative transfer modeling of various transitions of $\mathrm{CO}$ isotopologs.
\end{abstract}

Key words. ISM: general - ISM: individual objects: M8 - submillimeter: ISM - HII regions - ISM: clouds

\section{Introduction}

The influence of bright stars on their surrounding interstellar medium (ISM) is immense. Their strong ultraviolet and farultraviolet (FUV) fields give rise to bright HII regions and photodissociation regions (PDRs). These are the best grounds to study the effect of UV and FUV photons on the heating and chemistry of ISM. The fine structure lines of $\mathrm{C}^{+}$and $\mathrm{O}$, observable at far-infrared (FIR) wavelengths, are mainly responsible for the cooling in these regions (Tielens \& Hollenbach 1985b), which allow us to deduce the amount and sometimes the source of heating as well. The fine structure line of $\mathrm{C}^{+}$at $158 \mu \mathrm{m}$ is one of the brightest lines in PDRs and traces the transition from $\mathrm{H}^{+}$ to $\mathrm{H}$ and $\mathrm{H}_{2}$ as $\mathrm{C}$ has an ionization potential of $11.3 \mathrm{eV}$ (e.g., Pabst et al. 2017). In PDRs, a $C^{+}$layer extends to a depth of $A_{\mathrm{v}} \sim 2-4$, after which $\mathrm{C}^{+}$recombines to $\mathrm{C}$ probing the interface to CO (Hollenbach \& Tielens 1999). Deeper into the associated

\footnotetext{
* The reduced datacubes (FITS files) are only available at the CDS via anonymous ftp to cdsarc.u-strasbg. fr (130.79.128.5) or via http://cdsarc.u-strasbg.fr/viz-bin/qcat?J/A+A/615/A158

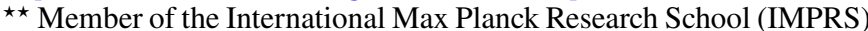
for Astronomy Astrophysics at the Universities of Bonn Cologne.
}

molecular clouds, cooling is dominated by the transitions of $\mathrm{CO}$, observable at (sub)millimeter and FIR wavelengths. Modeling the relative intensity distributions of multiple lines from various molecular and atomic species allows us to derive the physical conditions in PDRs.

Messier 8 (M8) is located in the Sagittarius-Carina arm, near our line of sight toward the Galactic center. It is located at a distance $\sim 1.25 \mathrm{kpc}$ ( $1^{\prime}$ corresponds to $0.36 \mathrm{pc}$ ) from the Sun (Damiani et al. 2004; Arias et al. 2006) with an error of $\sim 0.1 \mathrm{kpc}$ (Tothill et al. 2008) and is about $34 \times 12 \mathrm{pc}$ in diameter. M8 is associated with the open young stellar cluster NGC 6530, the HII region NGC 6523/33, and large quantities of molecular gas (Tothill et al. 2008).

The open cluster NGC 6530 (centered at RA $18^{\mathrm{h}} 04^{\mathrm{m}} 24^{\mathrm{s}}$, Dec $-24^{\circ} 21^{\prime} 12^{\prime \prime}(\mathrm{J} 2000)$ ) is a relatively young cluster (formed about 2-4 Myrs ago, Chen et al. 2007) and contains several bright O-type stars. The brightest among them is Her 36 (Woolf 1961) at RA $18^{\mathrm{h}} 03^{\mathrm{m}} 40^{\mathrm{s}} 3$, Dec $-24^{\circ} 22^{\prime} 43^{\prime \prime}(\mathrm{J} 2000)$. It is resolved into three main components: a close massive binary consisting of an $\mathrm{O} 9 \mathrm{~V}$ and a B0.5 V star and a more distant companion O7.5 V star (Arias et al. 2010; Sanchez-Bermudez et al. 2014). Her 36 is responsible for ionizing the gas in 


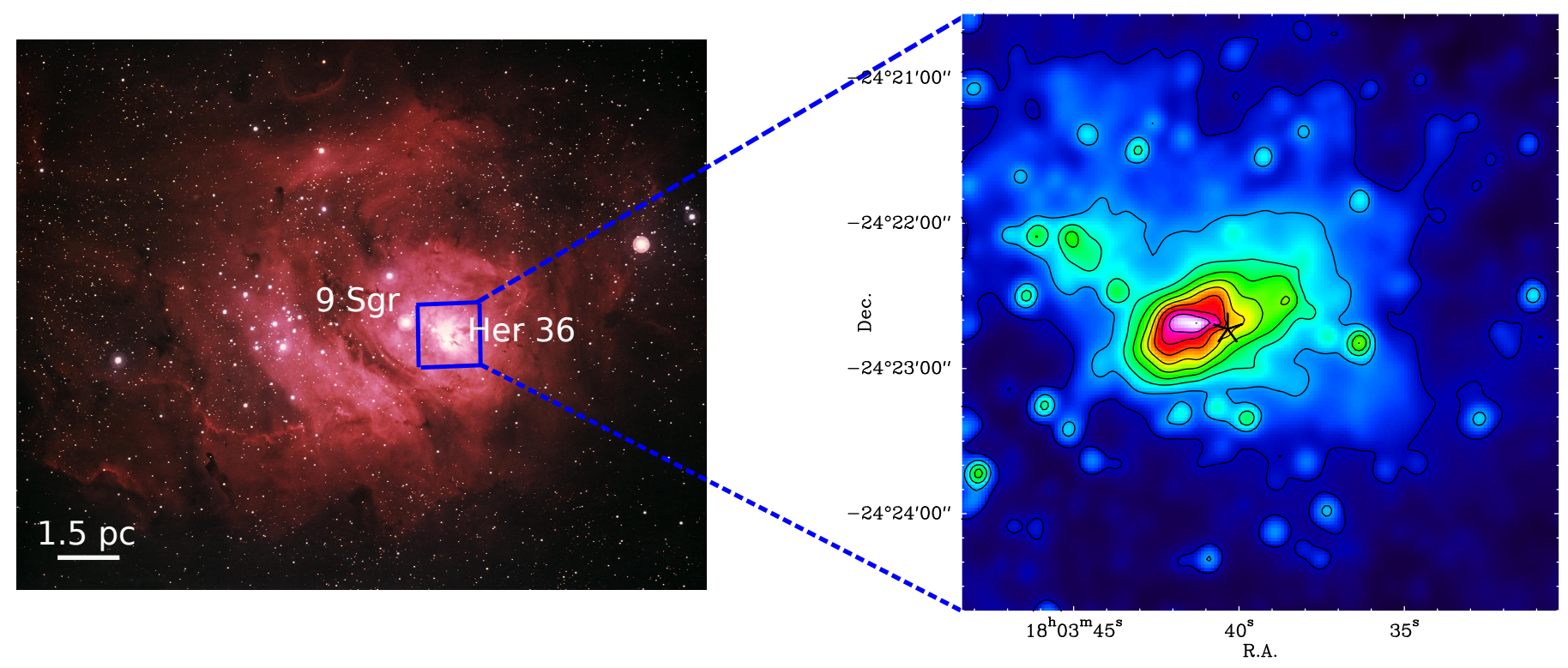

Fig. 1. Left panel: Lagoon Nebula, Messier object 8 (M8), or NGC 6523 in the constellation of Sagittarius, as seen by the Kitt Peak $4 \mathrm{~m}$ Mayall telescope in 1973. North is up, east to left. Credits to National Optical Astronomy Observatory/Association of Universities for Research in Astronomy/National Science Foundation Copyright WIYN Consortium, Inc., all rights reserved. Right panel: WISE $3.4 \mu \mathrm{m}$ image of the M8 region around Her 36 (denoted with a star) investigated in this paper. The contour levels are $10 \%$ to $100 \%$ in steps of $10 \%$ of the peak emission 4000 data number. The $1.5 \mathrm{pc}$ indicated in the lower left correspond to $4^{\prime}$.

the western half of the HII region of NGC 6523 including the bright Hourglass Nebula (Woolf 1961; Lada et al. 1976; Woodward et al. 1986). Lada et al. (1976) compared the optical and millimeter-wave observations of the M8 region and suggested the molecular cloud is located behind the HII region of the nebula, similar to the Orion-KL nebula. An ultracompact HII region, G5.97-1.17 is also very close to Her 36 at (RA $18^{\mathrm{h}} 03^{\mathrm{m}} 40.5^{\mathrm{s}}$, Dec $-24^{\circ} 22^{\prime} 44.3^{\prime \prime}(\mathrm{J} 2000)$ ) (Masqué et al. 2014).

A multiband near-IR image of Her 36 and its surroundings presented in Goto et al. (2006) shows the IR source Her 36 SE lying $0.25^{\prime \prime}$ SE of Her 36 and it is completely obscured. It is inferred to be an early-type B star with a visual extinction $A_{\mathrm{V}}>60$ mag that is deeply embedded in dense, warm dust and is powering the ultracompact HII region G5.97-1.17. The morphology of $\mathrm{H}_{2}$ and $\mathrm{CO} J=3 \rightarrow 2$ emission around Her 36 (White et al. 1997; Burton 2002) is in accordance with the Hubble Space Telescope (HST) jet-like feature detections extending 0.5" southeast of Her 36 (Stecklum et al. 1995), which suggest there might be a molecular outflow in the core of M8 (Burton 2002). X-ray emission from Her 36 and diffuse X-ray emission from the Hourglass region, which is the brightest part of the optically visible nebula located $\sim 15^{\prime \prime}$ east from Her 36 (Rauw et al. 2002), suggest the presence of a bubble of hot gas of size $0.4 \mathrm{pc}$ that is produced by the interaction of the stellar wind of Her 36 with the denser part of the molecular cloud in the background. Anomalously broad diffuse interstellar bands (DIBs) at 5780.5, 5797.1, 6196.0, and 6613.6 $\AA$ along with $\mathrm{CH}^{+}$and $\mathrm{CH}$ are found in absorption along the line of sight to Her 36 (Dahlstrom et al. 2013). $\mathrm{CH}^{+}$and $\mathrm{CH}$ are radiatively excited by strong FIR emission from the adjacent IR source Her 36 SE (Goto et al. 2006) and the broadening of DIBs is attributed to radiative pumping of closely spaced high- $J$ rotational levels of small polar carrier molecules (Dahlstrom et al. 2013; Oka et al. 2014; York et al. 2014). We performed (sub)millimeter observations related to these species that will be discussed in a future paper.

The eastern half of the HII region is illuminated by the $9 \mathrm{Sgr}$ stellar system, as shown in Fig. 1. $9 \mathrm{Sgr}$ is a well-known binary with an orbit of $\sim 9$ yr duration, consisting of an $\mathrm{O} 3.5 \mathrm{~V}$ primary and an O5-5.5 V secondary (Rauw et al. 2012). Southeast of the cluster core (NGC 6530), another cluster, M8E, although optically invisible, is associated with two massive star forming regions (Tothill et al. 2008). A superposition of four HII regions seems to be responsible for the ionization of the gas in M8: the Hourglass Nebula illuminated by Her 36, the core of NGC 6523 illuminated by Her 36, the remaining parts of NGC 6523 and NGC 6533 illuminated by 9 Sgr (O4V) (Tothill et al. 2008), and M8E illuminated by HD 165052 (Lynds \& Oneil 1982; Woodward et al. 1986).

Although M8 has been studied extensively in the X-ray, optical, and IR regimes (Stecklum et al. 1995; Damiani et al. 2004; Arias et al. 2006; Goto et al. 2006; Damiani et al. 2017), only few studies have been performed at millimeter and submillimeter wavelengths. White et al. (1997) reported the discovery of the second strongest source of millimeter and submillimeter wavelength $\mathrm{CO}$ line emission in our Galaxy toward Her 36 in M8 (White et al. 1997). Lada et al. (1976) compared optical and millimeter-wave observations to sketch the morphology of M8 where the core surrounding Her 36, the hourglass nebula with its structure and the eastern part of M8 are described. Tothill et al. (2002) presented submillimeter- and millimeter-wavelengths maps of the $J=2 \rightarrow 1$ and $J=3 \rightarrow 2$ transitions of ${ }^{12} \mathrm{CO}$ tracing the molecular gas and dust around Her 36.

We report a comprehensive survey of the $1.5 \times 1.5 \mathrm{pc}$ $\left(4^{\prime} \times 4^{\prime}\right)$ region around Her 36 (as shown by the blue square in Fig. 1) at FIR, millimeter- and submillimeter wavelengths to probe the physical conditions and image the morphology of this exceptional PDR. We present for the first time extended maps of this region in the $158 \mu \mathrm{m}$ fine structure line of $\mathrm{C}^{+}$, high- $J$ transitions of ${ }^{12} \mathrm{CO}$ emission observed with the GREAT ${ }^{1}$ receiver

1 GREAT is a development by the MPI für Radioastronomie and KOSMA/Universität zu Köln, in cooperation with the MPI für Sonnensystemforschung and the DLR Institut für Planetenforschung. 
M. Tiwari et al.: Unveiling the remarkable photodissociation region of Messier 8
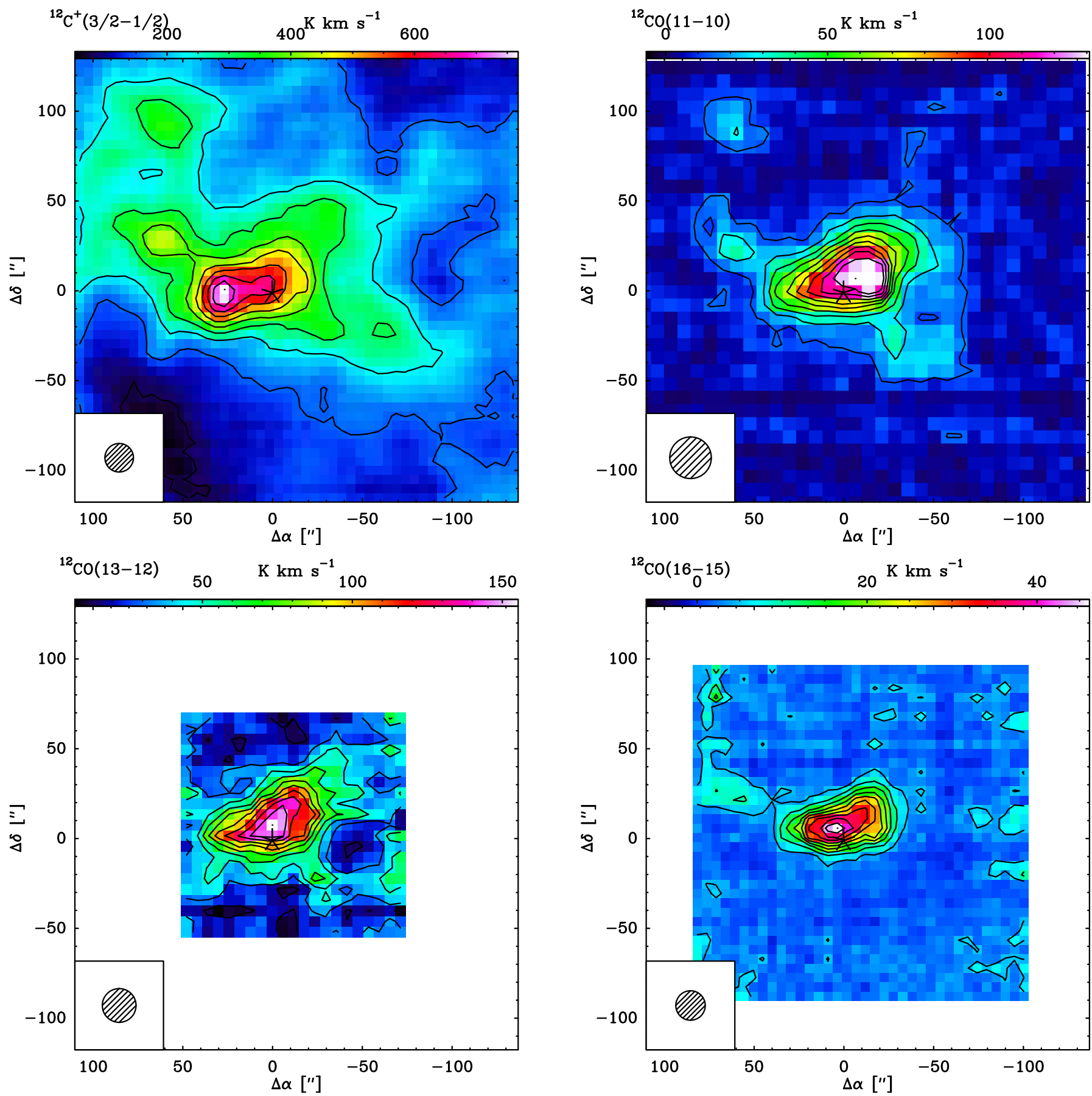

Fig. 2. Color maps of the integrated intensity of the [C II] $158 \mu \mathrm{m}$ and $J=11 \rightarrow 10, J=13 \rightarrow 12$, and $J=16 \rightarrow 15$ transitions of ${ }^{12} \mathrm{CO}$ toward Her 36, which is the central position $(\Delta \alpha=0, \Delta \delta=0)$ at $\mathrm{RA}(\mathrm{J} 2000)=18^{\mathrm{h}} 03^{\mathrm{m}} 40.3^{\mathrm{s}}$ and $\operatorname{Dec}(\mathrm{J} 2000)=-24^{\circ} 22^{\prime} 43^{\prime \prime}$, denoted with an asterisk. The contour levels are 10\% (>3 $\times$ rms, given in Table 1) to 100\% in steps of 10\% of the corresponding peak emission given in Table 1 . All maps are plotted using original beam sizes shown in the lower left of each map.

on board SOFIA observatory, the mid- $J$ transitions of ${ }^{12} \mathrm{CO}$ and ${ }^{13} \mathrm{CO}$ using the $\mathrm{PI} 230, \mathrm{FLASH}^{+}$, and $\mathrm{CHAMP}^{+}$receivers of the $\mathrm{APEX}^{2}$ telescope, and low- $J$ transitions of ${ }^{12} \mathrm{CO}$ and ${ }^{13} \mathrm{CO}$ using the EMIR receiver of the IRAM 30 m telescope.

\footnotetext{
2 This publication is based on data acquired with the Atacama Pathfinder EXperiment (APEX). APEX is a collaboration between the Max-Planck-Institut für Radioastronomie, the European Southern Observatory, and the Onsala Space Observatory.

3 Based on observations carried out with the IRAM $30 \mathrm{~m}$ telescope. IRAM is supported by INSU/CNRS (France), the MPG (Germany), and IGN (Spain).
}

\section{Observations}

\subsection{SOFIA/GREAT data}

The high- $J$ CO and $[\mathrm{C}$ II] $158 \mu \mathrm{m}$ observations summarized in Table 1 were conducted with the L1 channel of the German Receiver for Astronomy at Terahertz frequencies (GREAT; Heyminck et al. 2012) and the upGREAT LFA arrays (Risacher et al. 2016) on board the Stratospheric Observatory for Infrared Astronomy (SOFIA; Young et al. 2012). The data was acquired during observatory flight \#297 on 2016 May 14 at $14.2 \mathrm{~km}$ altitude and under a median water vapor column 
of $11 \mu \mathrm{m}$. The upGREAT was employed with 14 pixels (seven pixels for each polarization, with a hexagonal layout). The spectral analysis was performed by means of fast Fourier transform spectrometers (Klein et al. 2012), in a mode providing $4.0 \mathrm{GHz}$ bandwidth with $2^{14}$ spectral channels.

In the first setup we simultaneously mapped the ${ }^{12} \mathrm{CO}$ $J=11 \rightarrow 10$ transition at $1267.014 .486 \mathrm{GHz}$ and the $[\mathrm{C}$ II] ${ }^{2} P_{3 / 2} \rightarrow{ }^{2} P_{1 / 2}$ fine structure line at $1900.537 \mathrm{GHz}$. In a second setup the ${ }^{12} \mathrm{CO} J=13 \rightarrow 12$ and $J=16 \rightarrow 15$ transitions at $1496.923 \mathrm{GHz}$ and $1841.345 \mathrm{GHz}$ were mapped, respectively. Typical single-sideband system temperatures ranged between 1600 and $1800 \mathrm{~K}$ for the lower frequency L1 channel and between 2080 and $2260 \mathrm{~K}$ for the higher frequency upGREAT array with atmospheric transmissions of 0.90-0.94 and $0.85-0.88$, respectively. All maps shown in Fig. 2 were observed in on-the-fly (OTF) total power mode with a sampling of $6^{\prime \prime}$ centered on RA $18^{\mathrm{h}} 03^{\mathrm{m}} 40^{\mathrm{s}} .33$, Dec $-24^{\circ} 22^{\prime} 42^{\prime \prime} .7$ (J2000), the Her 36 location. We integrated 0.4 s per record for the $\mathrm{CO}(11 \rightarrow 10) /[\mathrm{CII}]$ setup, and $0.8 \mathrm{~s}$ for the $\mathrm{CO}$ $(13 \rightarrow 12) /(16 \rightarrow 15)$ setup. The originally chosen reference position at $(\Delta \alpha, \Delta \delta)=\left(+500^{\prime \prime},-500^{\prime \prime}\right)$ (relative to the map center) was found to be contaminated in both transitions of the first setup and was therefore changed in favor of a second, clean reference at offset $\left(+30^{\prime},-30^{\prime}\right)$, while the pointing accuracy of $<3^{\prime \prime}$ was maintained.

The raw data was calibrated with the program kalibrate (Guan et al. 2012), which is part of the KOSMA software package. The resulting antenna temperatures $T_{\mathrm{A}}^{*}$ were converted to main beam brightness temperatures $T_{\mathrm{mb}}=T_{\mathrm{A}}^{*} \cdot F_{\text {eff }} / B_{\text {eff }}$ using a forward efficiency $F_{\text {eff }}=0.97$ and beam efficiencies of 0.66 for the L1 channel of GREAT, and 0.58 to 0.68 for the upGREAT pixels, with a median value of 0.65 . In order to optimize the signal-to-noise ratio per channel, the spectra were smoothed to a resolution of $2.44 \mathrm{MHz}$, corresponding to $0.4 \mathrm{~km} \mathrm{~s}^{-1}$ for upGREAT and $0.6 \mathrm{~km} \mathrm{~s}^{-1}$ for the L1 channel. We subtracted spectral baselines of first order and subsequently produced data cubes with beam/3 sampling. For the beam sizes, see Table 1 .

\subsection{APEX data}

Observations of low- and mid- $J{ }^{12} \mathrm{CO}$ and ${ }^{13} \mathrm{CO}$ transitions were performed with APEX $12 \mathrm{~m}$ submillimeter telescope (Güsten et al. 2006) during 2015 June-August and 2016 July, September, and October. As shown in Table 1, we used the following receivers: PI230 to map the low- $J{ }^{12} \mathrm{CO}$ and ${ }^{13} \mathrm{CO}$ transitions, $\mathrm{FLASH}^{+}$in the 345 and $460 \mathrm{GHz}$ bands to map the mid- $J$ CO transitions, and $\mathrm{CHAMP}^{+}$in low- and high-frequency subarrays to map the higher frequency mid- $J \mathrm{CO}$ transitions.

We used the PI230 receiver to map $\mathrm{C}^{18} \mathrm{O} J=2 \rightarrow 1$ at $219.560 \mathrm{GHz},{ }^{13} \mathrm{CO} J=2 \rightarrow 1$ at $220.398 \mathrm{GHz}$, and ${ }^{12} \mathrm{CO} J=2 \rightarrow 1$ at $230.538 \mathrm{GHz}$. FLASH ${ }^{+}$was used in the $345 \mathrm{GHz}$ band to map the ${ }^{12} \mathrm{CO} J=3 \rightarrow 2$ transition at $345.795 \mathrm{GHz}$. FLASH ${ }^{+}$was also used in the $460 \mathrm{GHz}$ band to map the ${ }^{13} \mathrm{CO} \mathrm{J}=4 \rightarrow 3$ transition at $440.765 \mathrm{GHz}$, $\mathrm{C}^{18} \mathrm{O} J=4 \rightarrow 3$ at $439.088 \mathrm{GHz}$, and $\left[\mathrm{C}_{\mathrm{I}}\right]{ }^{3} P_{1} \rightarrow{ }^{3} P_{0}$ fine structure line at $492.160 \mathrm{GHz}$. The $\mathrm{CHAMP}^{+}$receiver was used to map ${ }^{12} \mathrm{CO} J=6 \rightarrow 5$ at $691.473 \mathrm{GHz}$ and ${ }^{13} \mathrm{CO} J=$ $6 \rightarrow 5$ at $661.067 \mathrm{GHz}$ in the low-frequency subarray complemented by ${ }^{12} \mathrm{CO} J=7 \rightarrow 6$ at $806.651 \mathrm{GHz}$ and ${ }^{13} \mathrm{CO} J=8 \rightarrow 7$ at $881.272 \mathrm{GHz}$ in the high-frequency subarray.

All maps shown in Fig. 3 were observed in OTF total power mode centered on RA $18^{\mathrm{h}} 03^{\mathrm{m}} 40.3$, Dec $-24^{\circ} 22^{\prime} 43^{\prime \prime}(\mathrm{J} 2000)$, which corresponds to the position of Her 36. The maps obtained from the observations carried out in July, September, and
October 2016 have a size of $240^{\prime \prime} \times 240^{\prime \prime}$. Maps that were obtained from the observations carried out in 2015 are comparatively smaller in size. We integrated $0.7 \mathrm{~s}$ per dump for all maps and enough coverages were performed to reach the rms noise levels as mentioned in Table 1 . The offset position relative to the center at $\left(30^{\prime},-30^{\prime}\right)$ was chosen as reference, similar to the SOFIA observations. The pointing accuracy $\left(<3^{\prime \prime}\right)$ was maintained by pointing at bright sources such as RAFGL5254 and $\mathrm{R}$ Dor every $1-1.5 \mathrm{~h}$. A forward efficiency $F_{\text {eff }}=0.95$ was used for all receivers, and the beam coupling efficiencies $B_{\text {eff }}=0.62,0.69,0.63,0.43$, and 0.32 were used for the PI230, $\mathrm{FLASH}^{+} 340, \mathrm{FLASH}^{+} 460, \mathrm{CHAMP}^{+} 660$, and $\mathrm{CHAMP}^{+} 810$ receivers, respectively.

\subsection{IRAM $30 \mathrm{~m}$ data}

Observations of low- $J{ }^{12} \mathrm{CO},{ }^{13} \mathrm{CO}$, and hydrogen recombination line observations were performed with the IRAM $30 \mathrm{~m}$ telescope in August 2016. We observed the whole $3 \mathrm{~mm}$ range using the EMIR receivers (Carter et al. 2012). We simultaneously mapped a region of $240^{\prime \prime} \times 240^{\prime \prime}$, which is similar to the size of most other maps previously observed with SOFIA and APEX in the ${ }^{12} \mathrm{CO}$ and ${ }^{13} \mathrm{CO} J=1 \rightarrow 0$ transitions (Table 1) and the hydrogen recombination lines $\mathrm{H} 40 \alpha$ to $\mathrm{H} 43 \alpha$ at 99.023 GHz, $92.034 \mathrm{GHz}, 85.688 \mathrm{GHz}$, and $79.912 \mathrm{GHz}$, respectively. Molecular high density tracers, which were also detected in our wide spectral band observation, will be analyzed in a subsequent paper.

All maps shown in Fig. 4 were observed in OTF total power mode centered on Her 36. Each subscan lasted $25 \mathrm{~s}$ and the integration time on the off-source reference position was $5 \mathrm{~s}$. The offset position relative to the center at $\left(30^{\prime},-30^{\prime}\right)$ was similar to that used for the SOFIA and APEX mapping and the pointing accuracy $\left(<3^{\prime \prime}\right)$ was maintained by pointing at the bright calibrator 1757-240 every 1-1.5 h. A forward efficiency $F_{\text {eff }}=0.95$ and a beam coupling efficiency $B_{\text {eff }}=0.69$ were adopted for the EMIR receivers. These values were taken from the latest (2015) commissioning report ${ }^{4}$.

All data reduction was performed using the CLASS and MIRA programs that are a part of the GILDAS ${ }^{5}$ software package and all the observations are summarized in Table 1.

\section{Results}

\subsection{Peak intensities of the molecular line emission}

The maxima of the distributions of the velocity integrated intensities of the emission in the $\left[\mathrm{C}_{\mathrm{I}}\right]$ and $\left[\mathrm{C}_{\mathrm{II}}\right]$ lines and different transitions of ${ }^{12} \mathrm{CO},{ }^{13} \mathrm{CO}$, and $\mathrm{C}^{18} \mathrm{O}$ are presented in Table 1. Figure 2 shows velocity integrated intensity maps of the ${ }^{12} \mathrm{CO}$ $J=11 \rightarrow 10,13 \rightarrow 12$, and $16 \rightarrow 15$ transitions. The emission in all the lines has a similar spatial distribution and peaks are found at about the same offset position $\left(\Delta \alpha=5.0^{\prime \prime}, \Delta \delta=5.0^{\prime \prime}\right)$ northwest of Her 36.

Figures 3 and 4 show velocity integrated intensity maps of low- and mid- $J$ transitions of ${ }^{12} \mathrm{CO},{ }^{13} \mathrm{CO}$, and $\mathrm{C}^{18} \mathrm{O}$, i.e., the $J=1 \rightarrow 0,2 \rightarrow 1,3 \rightarrow 2,6 \rightarrow 5,7 \rightarrow 6$ transitions of ${ }^{12} \mathrm{CO}$, the $J=1 \rightarrow 0,2 \rightarrow 1,4 \rightarrow 3$, and $6 \rightarrow 5$ transitions of ${ }^{13} \mathrm{CO}$, and the $J=1 \rightarrow 0$ transition of $\mathrm{C}^{18} \mathrm{O}$. The intensities of the low- $J$ transitions peak close to Her $36\left(\Delta \alpha=0.0^{\prime \prime}, \Delta \delta=0.0^{\prime \prime}\right)$ for ${ }^{12} \mathrm{CO}$ mid-J transitions; the peaks shift toward the northwest of Her 36 with offsets of $\left(\Delta \alpha=-13.0^{\prime \prime}, \Delta \delta=8.0^{\prime \prime}\right)$. It seems like there

\footnotetext{
WWW . iram. es/IRAMES/mainWiki/IRAM30mEfficiencies wWw . iram. fr/IRAMFR/GILDAS/
} 

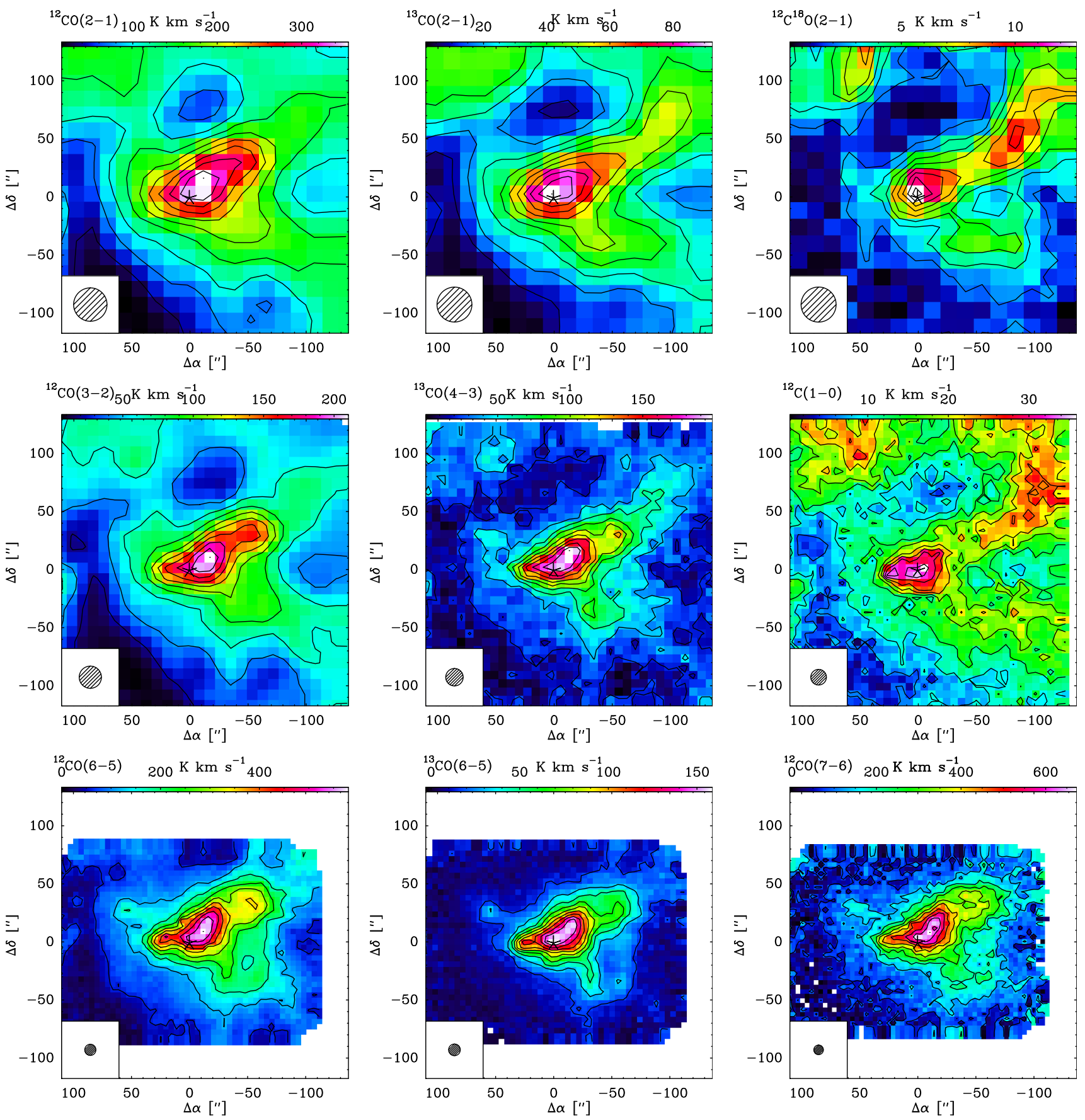

Fig. 3. Colour maps of the integrated intensity of the $J=2 \rightarrow 1, J=3 \rightarrow 2, J=6 \rightarrow 5$, and $J=7 \rightarrow 6$ transitions of ${ }^{12} \mathrm{CO}$, the $J=2 \rightarrow 1$, $J=4 \rightarrow 3$, and $J=6 \rightarrow 5$ transitions of ${ }^{13} \mathrm{CO}$, the $J=2 \rightarrow 1$ line of $\mathrm{C}^{18} \mathrm{O}$ and [C I] $1 \rightarrow 0$ toward Her 36 . This corresponds to the central position $(\Delta \alpha=0, \Delta \delta=0)$ at $\mathrm{RA}(\mathrm{J} 2000)=18^{\mathrm{h}} 03^{\mathrm{m}} 40.3^{\mathrm{s}}$ and $\operatorname{Dec}(\mathrm{J} 2000)=-24^{\circ} 22^{\prime} 43^{\prime \prime}$, denoted with an asterisk. The contour levels of ${ }^{12} \mathrm{C}^{18} \mathrm{O}$ and $[\mathrm{C} \mathrm{I}]$ are $3 \times \mathrm{rms}$ in steps of $2 \times \mathrm{rms}$, while those of other molecules are from 10\% ( $>3 \times \mathrm{rms}$, given in Table 1) to 100\% in steps of $10 \%$ of the corresponding peak emission given in Table 1. All maps are plotted using original beam sizes shown in the lower left of each map.

is a systematic shift in the peak emission of $\mathrm{CO}$ transitions with low- $J$ peaking near Her 36 , mid- $J$ peaking toward the northwest, while high- $J$ lines peak again closer to Her 36. Nevertheless, all maps show at least a small offset toward the northwest and the emission from $\mathrm{CO}$ transitions becomes more and more compact with increasing $J$.

Figures 2 and 3 show velocity integrated intensity maps of the $[\mathrm{C}$ II $]{ }^{2} P_{3 / 2} \rightarrow{ }^{2} P_{1 / 2}$ and $\left[\mathrm{C}_{\mathrm{I}}\right]{ }^{3} P_{1} \rightarrow{ }^{3} P_{0}$ transitions. [C I] peaks at Her 36 and is very bright toward the northwest of Her 36. [C II] peaks at an offset of $\left(\Delta \alpha=30.6^{\prime \prime}, \Delta \delta=-1.6^{\prime \prime}\right)$, which is toward the east of Her 36 and the emission extends even further. This extended emission comes from the part of the HII region that is illuminated by the stellar system 9 Sgr (Tothill et al. 2008).

Figure 4 shows a velocity integrated intensity map of an average of the $\mathrm{H} 40 \alpha$ to $\mathrm{H} 43 \alpha$ hydrogen recombination lines. We have taken the average to obtain a better signal-to-noise ratio. The distribution or the radio recombination line emission agrees well with the $5 \mathrm{GHz}$ continuum Very Large Array (VLA) interferometric map in Fig. 4 of Woodward et al. (1986) and the peak of 

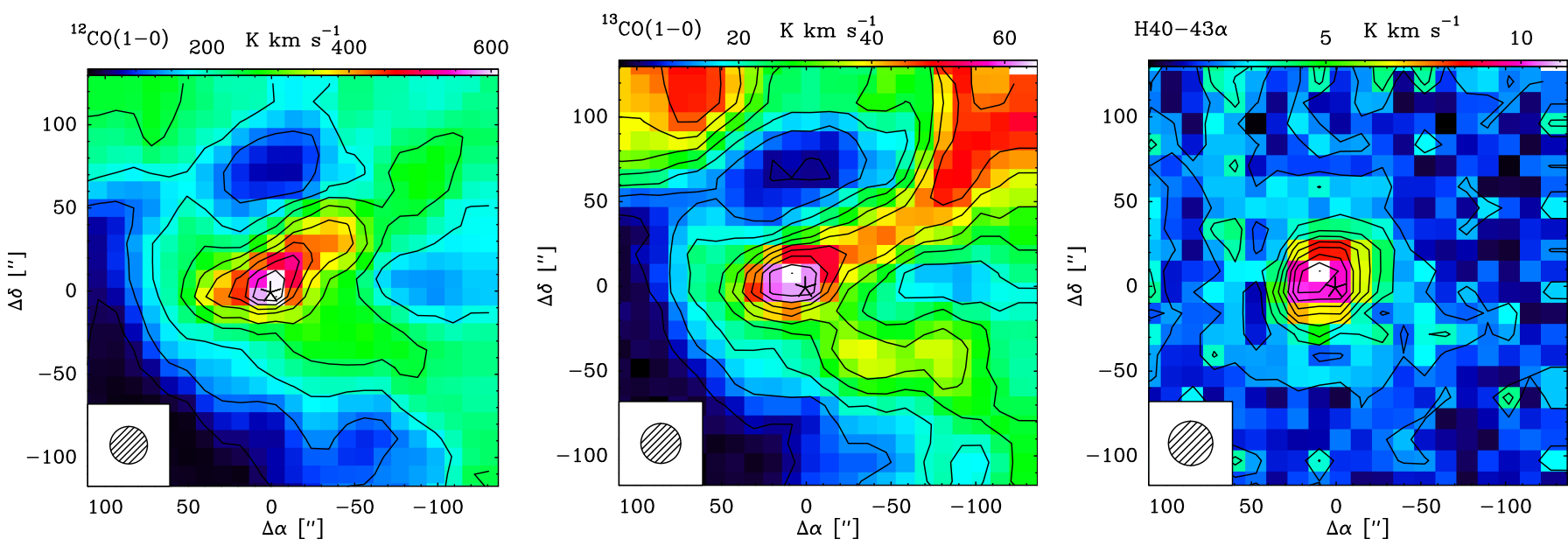

Fig. 4. Color maps of the integrated intensity (left to right) of the $J=1 \rightarrow 0$ transition of ${ }^{12} \mathrm{CO},{ }^{13} \mathrm{CO}$ and average of $\mathrm{H} \alpha 40,41,42$, and 43 lines toward Her 36, which is the central position $(\Delta \alpha=0, \Delta \delta=0)$ at $\mathrm{RA}(\mathrm{J} 2000)=18^{\mathrm{h}} 03^{\mathrm{m}} 40.3^{\mathrm{s}}$ and $\operatorname{Dec}(\mathrm{J} 2000)=-24^{\circ} 22^{\prime} 43^{\prime \prime}$, denoted with an asterisk. The contour levels are $10 \%$ ( $>3 \times$ rms, given in Table 1) to $100 \%$ in steps of $10 \%$ of the corresponding peak emission given in Table 1 . All maps are plotted using original beam sizes shown in the lower left of each map.

Table 1. Line parameters of observed transitions.

\begin{tabular}{|c|c|c|c|c|c|c|}
\hline Transition & Frequency $(\mathrm{GHz})$ & $\eta_{\mathrm{mb}}$ & $\theta_{\mathrm{mb}}\left({ }^{\prime \prime}\right)$ & Peak line flux $\left(\mathrm{K} \mathrm{km} \mathrm{s}^{-1}\right)$ & $\operatorname{rms}\left(\mathrm{K} \mathrm{km} \mathrm{s}^{-1}\right)$ & Telescope \\
\hline & & & ${ }^{12} \mathrm{CO}$ & & & \\
\hline$J=1 \rightarrow 0$ & 115.271 & 0.73 & 22.5 & 610.2 & 1.1 & IRAM 30 m/EMIR \\
\hline$J=2 \rightarrow 1$ & 230.538 & 0.65 & 28.7 & 355.5 & 0.4 & APEX/PI230 \\
\hline$J=3 \rightarrow 2$ & 345.796 & 0.73 & 19.2 & 210.2 & 0.8 & 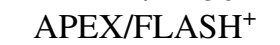 \\
\hline$J=6 \rightarrow 5$ & 691.473 & 0.43 & 9.6 & 580.1 & 4.0 & 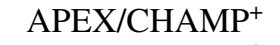 \\
\hline$J=7 \rightarrow 6$ & 806.652 & 0.34 & 8.2 & 673.4 & 19.0 & APEX/CHAMP ${ }^{+}$ \\
\hline$J=11 \rightarrow 10$ & 1267.014 & 0.68 & 22.9 & 130.9 & 3.5 & SOFIA/GREAT \\
\hline$J=13 \rightarrow 12$ & 1496.923 & 0.68 & 19.1 & 155.3 & 2.4 & SOFIA/GREAT \\
\hline \multirow[t]{2}{*}{$J=16 \rightarrow 15$} & 1841.346 & 0.70 & 14.8 & 46.2 & 1.8 & SOFIA/GREAT \\
\hline & & & ${ }^{13} \mathrm{CO}$ & & & \\
\hline$J=1 \rightarrow 0$ & 110.201 & 0.73 & 23.5 & 64.9 & 0.4 & IRAM 30 m/EMIR \\
\hline$J=2 \rightarrow 1$ & 220.399 & 0.65 & 30.1 & 92.6 & 0.6 & APEX/PI230 \\
\hline$J=4 \rightarrow 3$ & 440.765 & 0.59 & 15.0 & 198.1 & 2.5 & APEX/FLASH ${ }^{+}$ \\
\hline \multirow[t]{2}{*}{$J=6 \rightarrow 5$} & 661.067 & 0.45 & 10.0 & 158.8 & 3.2 & $\mathrm{APEX} / \mathrm{CHAMP}^{+}$ \\
\hline & & & $\mathrm{C}^{18} \mathrm{O}$ & & & \\
\hline \multirow[t]{2}{*}{$J=2 \rightarrow 1$} & 219.561 & 0.65 & 30.2 & 12.7 & 0.6 & APEX/PI230 \\
\hline & & & ${ }^{12} \mathrm{C}$ & & & \\
\hline \multirow[t]{2}{*}{${ }^{3} P_{1} \rightarrow{ }^{3} P_{0}$} & 492.160 & 0.59 & 13.5 & 34.0 & 1.8 & ${\mathrm{APEX} / \mathrm{FLASH}^{+}}^{+}$ \\
\hline & & & ${ }^{12} \mathrm{C}^{+}$ & & & \\
\hline \multirow[t]{2}{*}{${ }^{2} P_{3 / 2} \rightarrow{ }^{2} P_{1 / 2}$} & 1900.53 & 0.70 & 14.8 & 728.5 & 2.1 & SOFIA/GREAT \\
\hline & & & $\mathrm{H} \alpha$ & & & \\
\hline $\mathrm{H} 40-43 \alpha$ & $80-90$ & 0.73 & $\approx 26$ & 11.2 & 0.2 & IRAM $30 \mathrm{~m} / \mathrm{EMIR}$ \\
\hline
\end{tabular}

the $\mathrm{H} \alpha$ lines is at $\left(\Delta \alpha=10.0^{\prime \prime}, \Delta \delta=9.0^{\prime \prime}\right)$, close to the center of the Hourglass Nebula, which indicates the presence of hot ionized gas in the east of Her 36.

\subsection{Correlation between ${ }^{12} \mathrm{CO},{ }^{13} \mathrm{CO},[\mathrm{C} \mathrm{I}]$, and [C II]}

As can be seen from the velocity integrated intensity maps, emission from [C II] is spread out the most as compared to [C $\mathrm{I}],{ }^{12} \mathrm{CO}$, and ${ }^{13} \mathrm{CO}$. In order to visualize the correlation between these species, scatter plots of [C II] vs. [C I $]$, [C II] vs. ${ }^{12} \mathrm{CO} J=6 \rightarrow 5$, and [ $\mathrm{C}_{\mathrm{I}}$ ] vs. ${ }^{13} \mathrm{CO} J=2 \rightarrow 1$ are shown in Fig. 5. We chose $\mathrm{CO} 6 \rightarrow 5$ owing to its association with the warm PDR due to its higher upper level energy compared to low-J CO transitions, while we chose ${ }^{13} \mathrm{CO} 2 \rightarrow 1$ in particular, as its critical density is comparable to that of [C $\mathrm{I}]$. [C II] is correlated the least with ${ }^{12} \mathrm{CO} J=6 \rightarrow 5$. The Pearson correlation coefficient is $r=0.471$. Two branches appear to bud out in the upper left and lower right of this correlation. The upper left, where the $\left[\mathrm{C}\right.$ II] emission intensifies for a slowly strengthening ${ }^{12} \mathrm{CO} \mathrm{J}=$ $6 \rightarrow 5$ emission, corresponds to the northeast of Her 36 where 

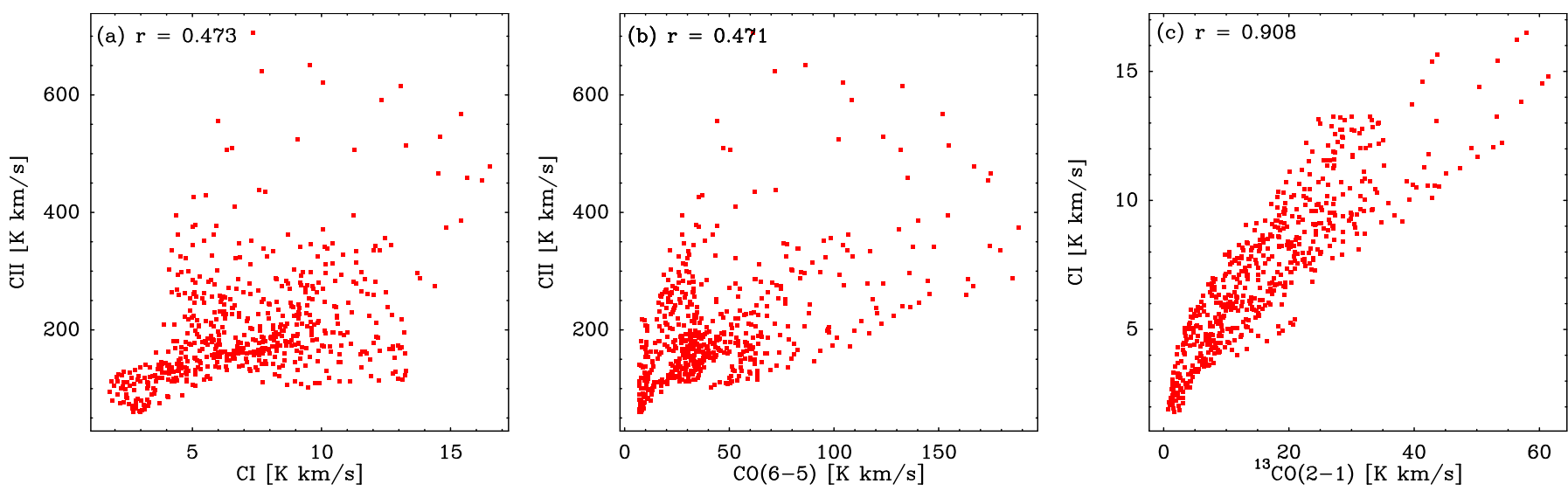

Fig. 5. Scatter plots and correlation coefficients $r$ between the velocity integrated intensity of $(a)[\mathrm{C}$ II] and $[\mathrm{C}$ I] $(b)[\mathrm{C}$ II $]$ and the $J=6 \rightarrow 5$ transition of ${ }^{12} \mathrm{CO}$, and $(c)\left[\mathrm{C}\right.$ I] and the $J=2 \rightarrow 1$ transition of ${ }^{13} \mathrm{CO}$. All data points were extracted from velocity integrated intensity maps convolved to the same beam size of $31^{\prime \prime}$.

Table 2. ${ }^{12} \mathrm{CO},{ }^{13} \mathrm{CO}$, [C I $]$, and [C II] line parameters.

\begin{tabular}{|c|c|c|c|}
\hline Offset $\left({ }^{\prime \prime}\right)^{a}$ & $V\left(\mathrm{~km} \mathrm{~s}^{-1}\right)$ & $\Delta V\left(\mathrm{~km} \mathrm{~s}^{-1}\right)$ & $T_{\text {peak }}^{b}(\mathrm{~K})$ \\
\hline \multicolumn{4}{|c|}{${ }^{12} \mathrm{CO} J=6 \rightarrow 5$} \\
\hline$(-40,35)$ & $10.33(0.06)$ & $3.62(0.16)$ & 77.24 \\
\hline \multirow{2}{*}{$(-13,8)$} & $5.92(0.10)$ & $3.30(0.26)$ & 58.41 \\
\hline & $10.43(0.04)$ & $2.92(0.10)$ & 134.43 \\
\hline \multirow{2}{*}{$(0,0)$} & $6.39(0.03)$ & $2.42(0.07)$ & 45.67 \\
\hline & $10.42(0.02)$ & $3.58(0.04)$ & 97.94 \\
\hline$(30,-2)$ & $10.28(0.09)$ & $4.73(0.20)$ & 62.60 \\
\hline$(60,27)$ & $11.49(0.07)$ & $2.51(0.17)$ & 53.77 \\
\hline \multicolumn{4}{|c|}{${ }^{13} \mathrm{CO} J=1 \rightarrow 0$} \\
\hline$(-40,35)$ & $8.47(0.04)$ & $3.00(0.11)$ & 8.26 \\
\hline$(-13,8)$ & $8.76(0.03)$ & $2.58(0.08)$ & 12.61 \\
\hline$(0,0)$ & $8.91(0.01)$ & $2.68(0.03)$ & 15.16 \\
\hline$(30,-2)$ & $9.37(0.03)$ & $2.48(0.09)$ & 11.15 \\
\hline$(60,27)$ & $10.48(0.02)$ & $1.45(0.05)$ & 8.46 \\
\hline \multicolumn{4}{|c|}{${ }^{12} \mathrm{C}^{3} P_{1} \rightarrow{ }^{3} P_{0}$} \\
\hline$(-40,35)$ & $9.40(0.63)$ & $5.91(2.16)$ & 2.84 \\
\hline$(-13,8)$ & $9.47(0.25)$ & $3.63(0.63)$ & 5.62 \\
\hline$(0,0)$ & $9.89(0.18)$ & $3.86(0.47)$ & 7.65 \\
\hline$(30,-2)$ & $10.05(0.30)$ & $4.01(0.64)$ & 5.45 \\
\hline$(60,27)$ & $11.80(0.09)$ & $0.68(0.35)$ & 5.85 \\
\hline \multicolumn{4}{|c|}{${ }^{12} \mathrm{C}^{+2} P_{3 / 2} \rightarrow{ }^{2} P_{1 / 2}$} \\
\hline \multirow{2}{*}{$(-40,35)$} & $5.40(0.14)$ & $2.84(0.30)$ & 33.45 \\
\hline & $9.76(0.11)$ & $3.80(0.31)$ & 45.05 \\
\hline \multirow{2}{*}{$(-13,8)$} & $4.92(0.10)$ & $3.18(0.25)$ & 43.04 \\
\hline & $9.71(0.04)$ & $2.89(0.10)$ & 110.50 \\
\hline \multirow{2}{*}{$(0,0)$} & $5.12(0.23)$ & $3.31(0.57)$ & 24.97 \\
\hline & $9.94(0.06)$ & $4.11(0.14)$ & 114.49 \\
\hline \multirow{2}{*}{$(30,-2)$} & $3.88(0.16)$ & $4.01(0.36)$ & 37.66 \\
\hline & $9.72(0.07)$ & $4.86(0.16)$ & 100.10 \\
\hline$(60,27)$ & $10.22(0.04)$ & $3.34(0.09)$ & 112.83 \\
\hline
\end{tabular}

Notes. ${ }^{(a)}$ The reference position is that of Her $36 .{ }^{(b)}$ In units of main beam brightness temperature.

$\left[\mathrm{C}\right.$ II] is more extended. The lower right, where ${ }^{12} \mathrm{CO} J=6 \rightarrow 5$ emission intensifies at a faster rate than $[\mathrm{C} \mathrm{II}]$, corresponds to the southwest of Her 36 , where ${ }^{12} \mathrm{CO} J=6 \rightarrow 5$ is much more prominent. The correlation of $[\mathrm{C} \mathrm{II}]$ with $[\mathrm{C} \mathrm{I}]$ has a correlation coefficient of $r=0.473$ and again shows two different branches corresponding to different regions. The upper left, where [C II] emission gets brighter for an almost constant [ $\left.\mathrm{C}_{\mathrm{I}}\right]$ emission, corresponds to the northeast of Her 36. The branch in the lower right, similar to the situation shown in Fig. 5b, corresponds to the southwest of Her 36. In contrast to these correlations, $\left[\mathrm{C}_{\mathrm{I}}\right]$ is well correlated with ${ }^{13} \mathrm{CO} J=2 \rightarrow 1$ with $r=0.908$. This resembles the case M17 SW, for which a correlation coefficient of [C $\mathrm{I}$ ] with ${ }^{13} \mathrm{CO} J=2 \rightarrow 1$ was reported to be 0.942 (Pérez-Beaupuits et al. 2015c).

\subsection{Channel maps}

In order to investigate the differences in the distribution of ionized and atomic carbon, channel maps of the ${ }^{2} P_{3 / 2} \rightarrow{ }^{2} P_{1 / 2}$ transition of [C II] are compared to those of the $\left[\mathrm{C}_{\mathrm{I}}\right]^{3} P_{1} \rightarrow{ }^{3} P_{0}$ transition. Figure 6 shows that the emission from [C II] (lower panels) is more spread out as compared to that from [ $\left.\mathrm{C}_{\mathrm{I}}\right]$ (upper panels). In the velocity range from 2 to $6 \mathrm{~km} \mathrm{~s}^{-1}$ and 15 to $17 \mathrm{~km} \mathrm{~s}^{-1}$ there is no emission from [C $\mathrm{I}$ ] while there is emission from $\left[\mathrm{C}_{\mathrm{II}}\right]$ close to Her 36 and toward the east of it, respectively. In the range from 7 to $9 \mathrm{~km} \mathrm{~s}^{-1}$ both [C II] and [C I] emission are found toward the west. These structures extend further toward the northeast for higher velocities in the range of 12 to $15 \mathrm{~km} \mathrm{~s}^{-1}$. This is very similar to the case of M17 SW, where the $[\mathrm{C}$ II] channel map shows a strong spatial association with [C I] and CO channel maps only at intermediate 10 to $24 \mathrm{~km} \mathrm{~s}^{-1}$ velocities. While at lower $\left(<10 \mathrm{~km} \mathrm{~s}^{-1}\right)$ and higher $\left(>24 \mathrm{~km} \mathrm{~s}^{-1}\right)$ velocity channels, [C II] emission is mostly not associated with the other tracers of dense and diffuse gas (Pérez-Beaupuits et al. 2015c). Notably, our [C II] channel maps show a clumpy structure at an offset of $\left(\Delta \alpha=60.0^{\prime \prime}, \Delta \delta=27.0^{\prime \prime}\right)$ which is missing in the $\left[\mathrm{C}_{\mathrm{I}}\right]$ maps; this complements the argument that the east of Her 36 is comprised of hot gas and strong UV fields capable of ionizing carbon, i.e., it is part of an HII region. This is consistent with the $\mathrm{H} \alpha$ and $5 \mathrm{GHz}$ continuum VLA interferometric maps presented by Woodward et al. (1986) in their Figs. 1 and 4, which also have their peak intensities east from Her 36.

\subsection{Ancillary data}

For a multiwavelength view of M8 and in order to relate our observations to the dense and cold molecular cloud and the hot ionized gas in M8, we compared our data with observations obtained at other wavelength ranges. The surveys chosen 

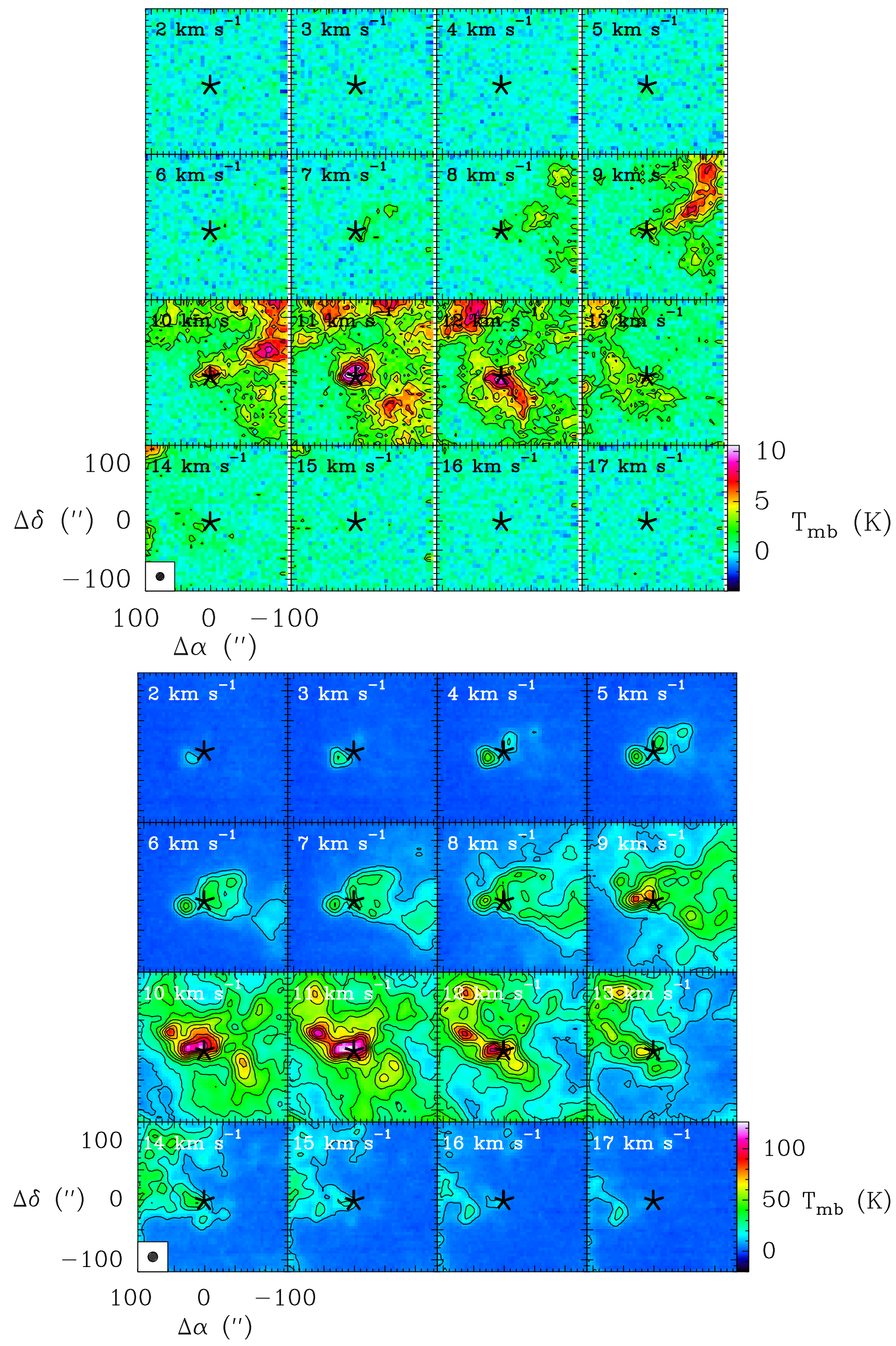

Fig. 6. Velocity channel maps of the ${ }^{3} P_{1} \rightarrow{ }^{3} P_{0}$ transition of [C I] (upper 16 panels) and the ${ }^{2} P_{3 / 2} \rightarrow{ }^{2} P_{1 / 2}$ transition of [C II] (lower 16 panels) in a range of $2-17 \mathrm{~km} \mathrm{~s}^{-1}$ with a channel width of $1 \mathrm{~km} \mathrm{~s}^{-1}$ toward Her 36 , which is the central position $(\Delta \alpha=0, \Delta \delta=0)$ at $\mathrm{RA}(\mathrm{J} 2000)=18^{\mathrm{h}} 03^{\mathrm{m}} 40.3^{\mathrm{s}}$ and $\operatorname{Dec}(\mathrm{J} 2000)=-24^{\circ} 22^{\prime} 43^{\prime \prime}$, denoted with a black asterisk. The contour levels of [C I] are $3 \times$ rms in steps of $2 \times$ rms, while those of [C II] are from $10 \%(>3 \times \mathrm{rms})$ to $100 \%$ in steps of $10 \%$ of the corresponding peak emission. All maps are plotted using original beam sizes shown in the lower left of both panels. 

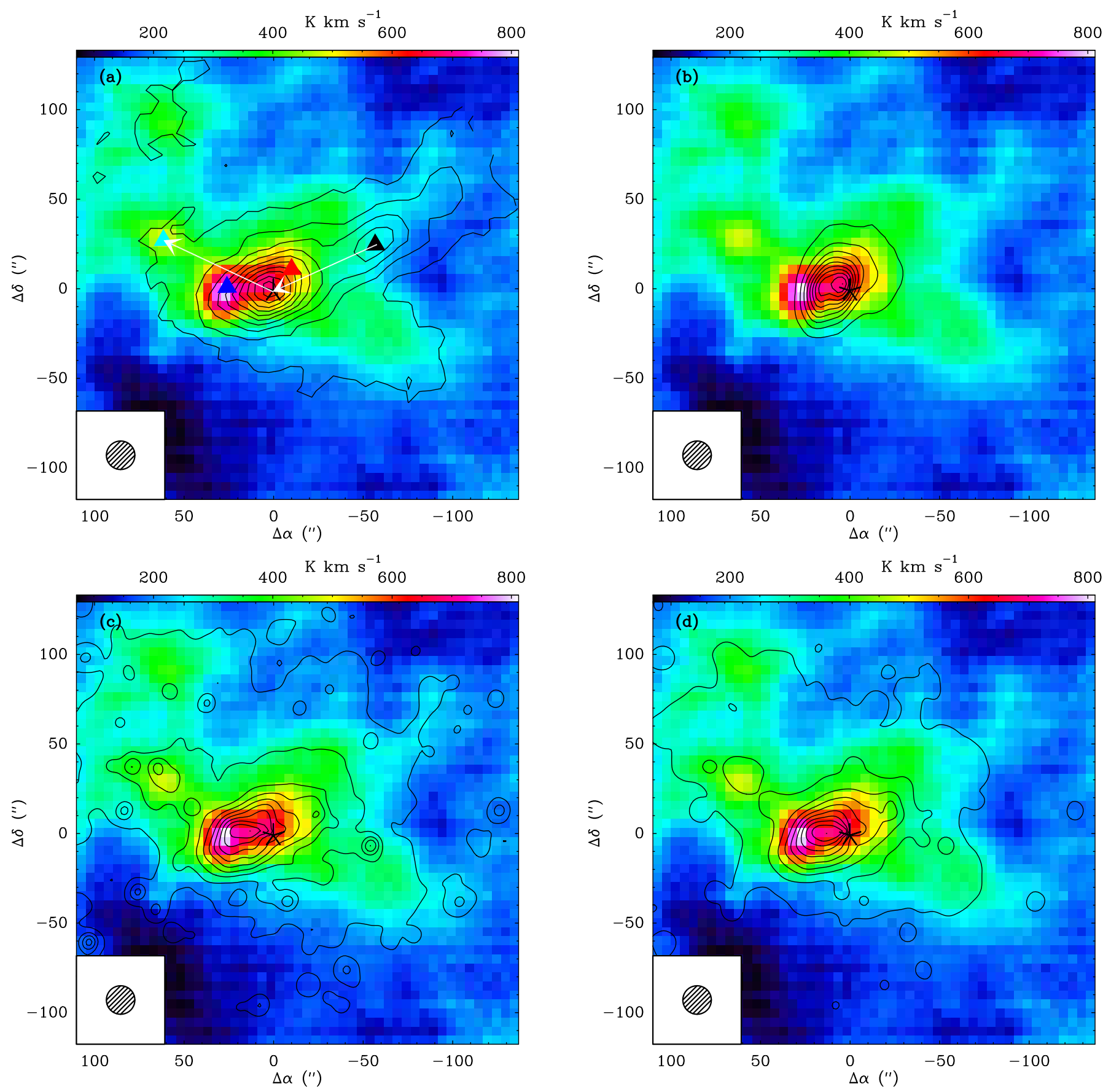

Fig. 7. A [C II] velocity integrated intensity map overlaid with contours of (a) ATLASGAL $870 \mu$ m continuum emission. Important offset positions discussed in the text are indicated with triangles of different colors: black represents the secondary ATLASGAL peak deep inside the molecular cloud at $\left(\Delta \alpha=-53.0^{\prime \prime}, \Delta \delta=23.0^{\prime \prime}\right)$, red represents the emission peak of mid- $J$ transitions of ${ }^{12} \mathrm{CO}$ and ${ }^{13} \mathrm{CO}$ at $\left(\Delta \alpha=-13.0^{\prime \prime}, \Delta \delta=8.0^{\prime \prime}\right)$, blue represents the peak of the [C II] $158 \mu \mathrm{m}$ emission at $\left(\Delta \alpha=30.0^{\prime \prime}, \Delta \delta=-2.0^{\prime \prime}\right)$, and light blue represents the clump to the east of Her 36 observed in the channel maps of [C II] at $\left(\Delta \alpha=60.0^{\prime \prime}, \Delta \delta=27.0^{\prime \prime}\right)$. The white arrows point along the molecular cloud in the west to the HII regions in the east of Her 36; (b) VLA $1.3 \mathrm{~cm}$ free-free continuum emission; (c) WISE $3.4 \mu \mathrm{m}$, and $(d)$ WISE $4.6 \mu$ m mid-infrared continuum. Her 36 is the central position denoted with an asterisk. The contour levels are 5\% to $95 \%$ in steps of $10 \%$ of the peak emission for $(a)$ and $10 \%$ to $100 \%$ in steps of $10 \%$ of the peak emission for $(b),(c)$, and $(d)$.

for this comparison are as follows: firstly, we extracted data from the $870 \mu \mathrm{m}$ APEX Telescope Large Area Survey of the Galaxy (ATLASGAL; Schuller et al. 2009) performed with the APEX $12 \mathrm{~m}$ telescope using the Large APEX BOlometer CAmera (LABOCA). The dust continuum emission probes dense and cold clumps in the ISM of our Galaxy. Figure 7a shows our $[\mathrm{C}$ II] velocity integrated intensity map overlaid with the ATLASGAL dust continuum image that peaks at Her 36 and also traces the cold molecular cloud in the northwest. The dust emission morphology is similar to the ${ }^{12} \mathrm{CO},{ }^{13} \mathrm{CO}$, and $\mathrm{C}^{18} \mathrm{O}$ distribution. Secondly, we used data from the National Radio Astronomy Observatory (NRAO)/Very Large Array (VLA) Archive Survey $\left(\mathrm{NVAS}^{6}\right)$. Figure $7 \mathrm{~b}$ shows the $\left[\mathrm{C}_{\mathrm{II}}\right]$ velocity integrated intensity map overlaid with the $1.3 \mathrm{~cm}$ radio

6 http://archive.nrao.edu/nvas/ 

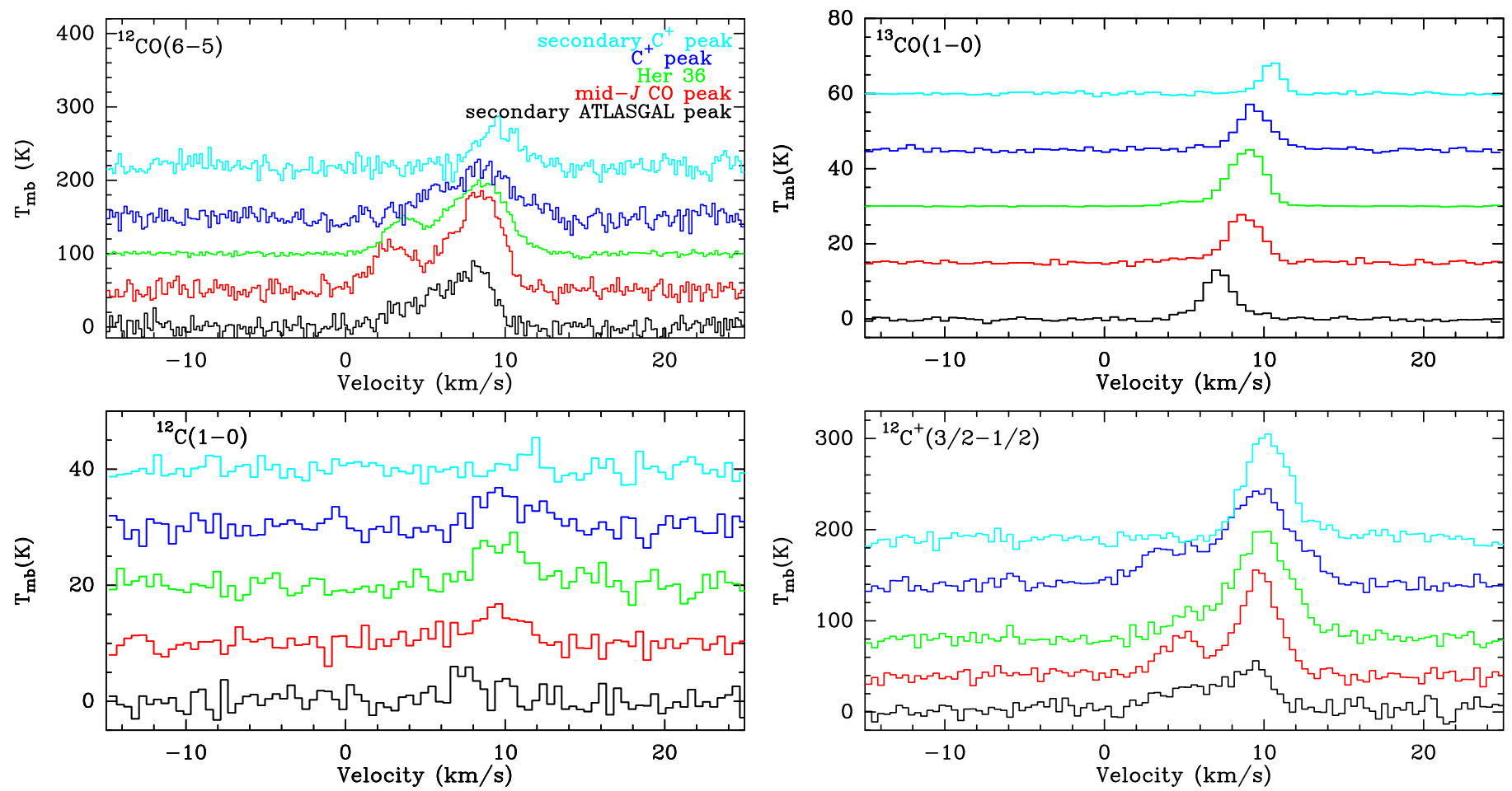

Fig. 8. Line profiles at different offsets (") relative to Her 36 are shown in different colors at five positions mentioned in the upper left plot. For the detailed positions, see Sect. 3.5. Upper panels: $\mathrm{J}=6 \rightarrow 5{ }^{12} \mathrm{CO}$ and $\mathrm{J}=1 \rightarrow 0{ }^{13} \mathrm{CO}$ spectra; lower panels: ${ }^{3} P_{1} \rightarrow{ }^{3} P_{0}$ and ${ }^{2} P_{3 / 2} \rightarrow{ }^{2} P_{1 / 2}$ transitions of $[\mathrm{C} \mathrm{I}]$ and $[\mathrm{C} \mathrm{II}]$. All spectra were extracted from their original beam sizes as mentioned in Table 1.

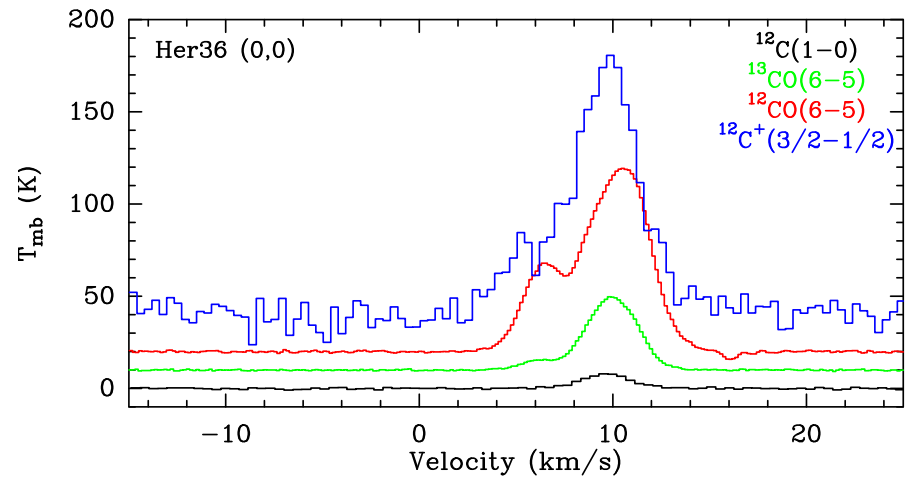

Fig. 9. Line profiles toward Her 36 for $[\mathrm{C} \mathrm{I}]{ }^{3} P_{1} \rightarrow{ }^{3} P_{0},{ }^{13} \mathrm{CO} J=6 \rightarrow 5$, ${ }^{12} \mathrm{CO} J=6 \rightarrow 5$, and $[\mathrm{C} \mathrm{II}]{ }^{2} P_{3 / 2} \rightarrow{ }^{2} P_{1 / 2}$. All spectra are extracted from maps that were convolved to the same beam size of $15^{\prime \prime}$.

continuum image ${ }^{7}$, which peaks very close to Her 36 and traces free-free emission from the HII region NGC 6523/33. This compact $\mathrm{HII}$ region, which is also traced by the $\mathrm{H}$ recombination lines with the IRAM $30 \mathrm{~m}$ telescope, is also shown in Fig. 4 (right panel). Thirdly, the Wide-field Infrared Survey Explorer (WISE) imaged the sky at four mid-infrared wavelengths. Figure $7 \mathrm{c}$ and $\mathrm{d}$ compare the $[\mathrm{C} \mathrm{II}]$ velocity integrated intensity map with WISE $3.4 \mu \mathrm{m}$ (band 1) and $4.6 \mu \mathrm{m}$ (band 2) continuum images, which peak closer to the [C II] peak. Overall, the mid-infrared emission that originates from hot dust shows the best agreement with the morphology seen in the $[\mathrm{C}$ II] image; both probe hot material from HII regions and warm surfaces of PDRs.

7 NRAO/VLA Archive Survey, (c) 2005-2007 AUI/NRAO.

\subsection{Spectra of ${ }^{12} \mathrm{CO},{ }^{13} \mathrm{CO},[\mathrm{C} \mathrm{I}]$ and [C II] emission lines at different offsets}

Figure 8 shows a comparison between the spectra of ${ }^{12} \mathrm{CO}$, ${ }^{13} \mathrm{CO},\left[\mathrm{C}_{\mathrm{I}}\right]$, and $[\mathrm{C} \mathrm{II}]$ emission lines at different offsets relative to Her 36. Line parameters of Gaussian fits to profiles are reported in Table 2. In several cases the profiles show evidence of two velocity components that were fit separately. The ${ }^{12} \mathrm{CO}$ $J=6 \rightarrow 5$ and ${ }^{13} \mathrm{CO} J=1 \rightarrow 0$ transitions are representative of the general appearance of all ${ }^{12} \mathrm{CO}$ and ${ }^{13} \mathrm{CO}$ line profiles discussed in this paper. The different offsets were chosen along a curved line from the molecular cloud in the west to the east of Her 36 (see Fig. 7a): the secondary $\mathrm{C}^{+}$peak, which corresponds to the clump observed in the channel map of [C II] at $\left(\Delta \alpha=60.0^{\prime \prime}, \Delta \delta=27.0^{\prime \prime}\right)$; the $\mathrm{C}^{+}$peak, which is the emission peak of the ${ }^{2} P_{3 / 2} \rightarrow{ }^{2} P_{1 / 2}$ transition of $\left[\mathrm{C}\right.$ II] at $\left(\Delta \alpha=30.0^{\prime \prime}\right.$, $\left.\Delta \delta=-2.0^{\prime \prime}\right)$; Her 36 is located at $\left(\Delta \alpha=0.0^{\prime \prime}, \Delta \delta=0.0^{\prime \prime}\right)$; the mid- $J$ CO peak, which is the mid- $J$ transition emission peak of ${ }^{12} \mathrm{CO}$ and ${ }^{13} \mathrm{CO}$ at $\left(\Delta \alpha=-13.0^{\prime \prime}, \Delta \delta=8.0^{\prime \prime}\right)$; and the secondary ATLASGAL peak arises from deep into the molecular cloud to the west traced by ATLASGAL at $\left(\Delta \alpha=-53.0^{\prime \prime}\right.$, $\left.\Delta \delta=23.0^{\prime \prime}\right)$.

A lower velocity $\left(2-6 \mathrm{~km} \mathrm{~s}^{-1}\right)$ component is spectrally resolved at several positions. The higher velocity component emission lines have blue-shifted wings in the molecular cloud in the west, while the emission is red-shifted toward the [C II] peak toward the east compared to their emission peaking at $9 \mathrm{~km} \mathrm{~s}^{-1}$ toward our reference position, Her 36. Furthermore, the peak of the lines shifts from the east to the west to lower velocities. The ${ }^{12} \mathrm{CO}$ and ${ }^{13} \mathrm{CO}$ line profiles are similar in being most intense with broadest line widths at the mid- $J$ transition emission peak of ${ }^{12} \mathrm{CO}$ and ${ }^{13} \mathrm{CO}\left(\Delta \alpha=-13.0^{\prime \prime}, \Delta \delta=8.0^{\prime \prime}\right)$ and at Her 36 itself, while getting less intense with narrower line widths at the 

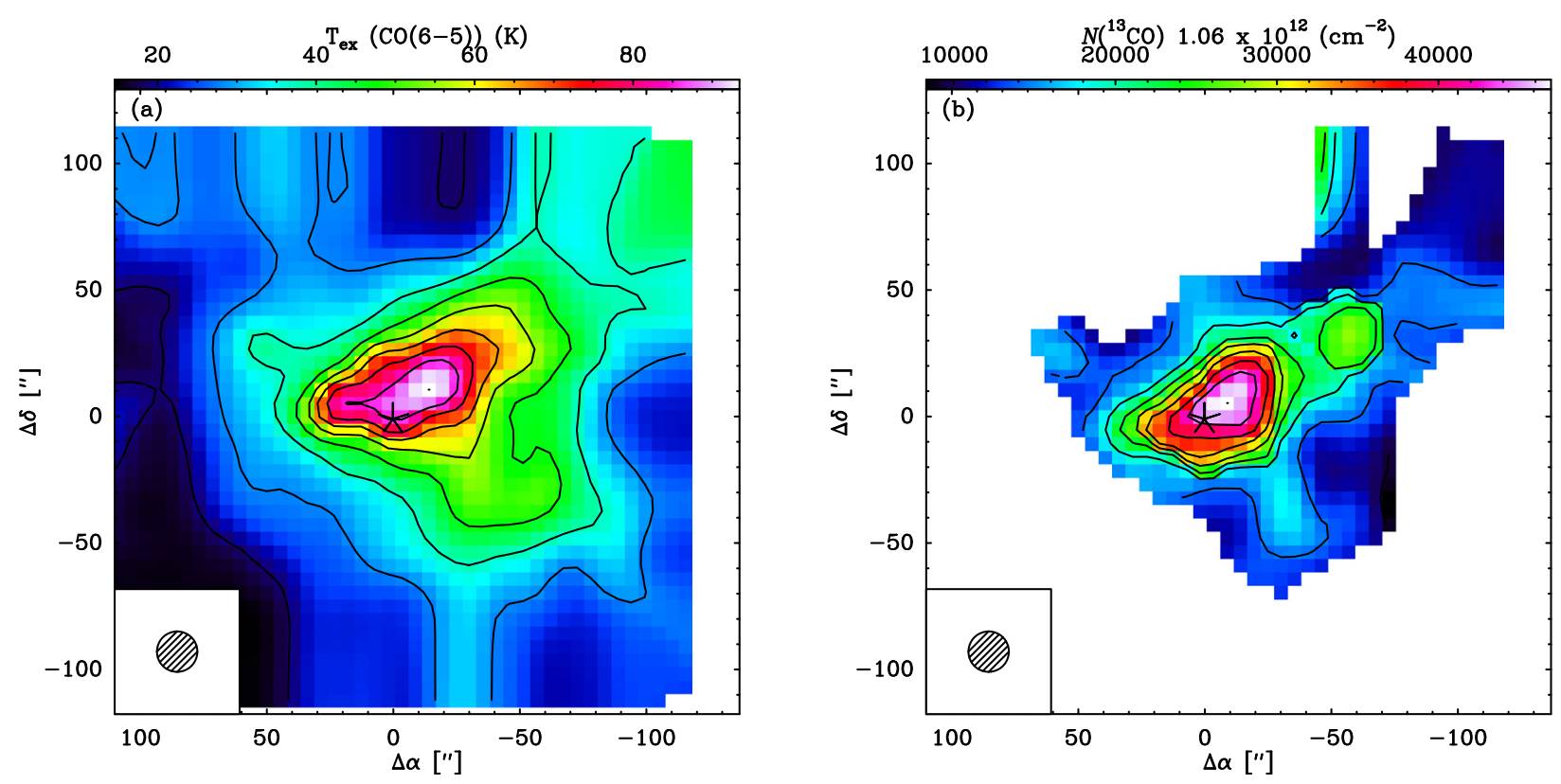

Fig. 10. For the $J=6 \rightarrow 5$ transition (a) shows the excitation temperature of ${ }^{12} \mathrm{CO}$, which is assumed to be equal to that of ${ }^{13} \mathrm{CO}$ and $(b)$ shows the column density of ${ }^{13} \mathrm{CO}$. The asterisk represents Her 36, which is the central position $(\Delta \alpha=0, \Delta \delta=0)$ at $\mathrm{RA}(\mathrm{J} 2000)=18^{\mathrm{h}} 03^{\mathrm{m}} 40.3^{\mathrm{s}}$ and $\operatorname{Dec}(\mathrm{J} 2000)=-24^{\circ} 22^{\prime} 43^{\prime \prime}$. The contour levels are $10 \%$ to $100 \%$ in steps of $10 \%$ of the corresponding peak emissions. All maps are plotted using original beam sizes shown in the lower left of each map.

[C II] peak. The $\left[\mathrm{C}_{\mathrm{I}}\right]$ line profile gets most intense with broadest line width toward Her 36 itself with almost no emission from the clumpy structure in the HII region at an offset of $\left(\Delta \alpha=60.0^{\prime \prime}\right.$, $\left.\Delta \delta=27.0^{\prime \prime}\right)$. As can also be seen from the comparison of various molecular transitions at Her 36 in Fig. 9, $\mathrm{CO}$ and [C $\mathrm{I}]$ are not associated with $[\mathrm{C}$ II] at lower and higher velocities (see also Figs. 5 and 6). This is very similar to M17 SW as reported by Pérez-Beaupuits et al. (2015c), where [C II] is not associated with other gas tracers at lower and higher velocities.

\section{Analysis}

In this section we determine the temperature and density in the PDR of M8 with several complementary methods. We start by using the data for the $J=6 \rightarrow 5$ transition of $\mathrm{CO}$, which has the highest angular resolution, to estimate excitation temperatures and column densities throughout the PDR as probed by the mid$J$ CO emission.

\subsection{Excitation temperature and column density estimates}

A detailed description of spectral line radiative transfer relevant here can be found in Draine (2011) and Mangum \& Shirley (2016). For a constant excitation temperature $T_{\mathrm{ex}}$, we can integrate the radiative transfer equation to obtain the observable Rayleigh-Jeans equivalent temperature $T_{\mathrm{R}}^{*}$ (Eq. (1) in Peng et al. 2012). The background radiation temperature comprises the cosmic background radiation of $2.73 \mathrm{~K}$ and the radiation from warm dust. The latter was calculated using the results from the Spectral and Photometric Imaging Receiver (SPIRE) of the European Space Agency (ESA) Herschel Space observatory. Data obtained in the second band at $350 \mu \mathrm{m}$ (close to the ${ }^{12} \mathrm{CO} 7 \rightarrow 6$ line) wavelength was used. The maximum intensity in the analyzed region around Her 36 is about $1200 \mathrm{MJy} \mathrm{sr}^{-1}$ near the dense molecular cloud, which corresponds to a RayleighJeans equivalent brightness temperature of about $0.06 \mathrm{~K}$ from dust. Thus, the total contribution from dust and background can be neglected as it contributes $\leq 1 \%$ to the resulting $T_{\mathrm{R}}^{*}$.

Assuming that the excitation temperature for ${ }^{12} \mathrm{CO}$ and ${ }^{13} \mathrm{CO}$ is the same and ${ }^{12} \mathrm{CO}$ is optically thick,

$\frac{T_{\mathrm{R}}^{*}\left({ }^{12} \mathrm{CO}\right)}{T_{\mathrm{R}}^{*}\left({ }^{13} \mathrm{CO}\right)}=\frac{1}{1-e^{-\tau\left({ }^{13} \mathrm{CO}\right)}}$.

The excitation temperature of the ${ }^{12} \mathrm{CO} J=6 \rightarrow 5$ transition can be estimated by further assuming a beam filling factor of unity, i.e., $T_{\mathrm{R}}^{*}=T_{\mathrm{MB}}$. This equation is written as

$T_{\mathrm{ex}}=33.2\left[\ln \left(1+\frac{33.2}{T_{\mathrm{MB}}\left({ }^{12} \mathrm{CO}\right)}\right)\right]^{-1} \mathrm{~K}$,

where $T_{\mathrm{MB}}$ is the main beam brightness temperature in $\mathrm{K}$ that is estimated from the peak temperature map of ${ }^{12} \mathrm{CO} \mathrm{J}=$ $6 \rightarrow 5$ transition. The resulting $T_{\mathrm{ex}}$ distribution is shown in Fig. 10a. Formally, we show lower limits to the excitation temperature due to the assumption of a beam filling factor of unity. It is highest immediately in the northwest of Her 36 and decreases with distance from the star.

Using the computed $T_{\mathrm{ex}}$ and the main beam brightness temperature $T_{\mathrm{MB}}$ estimated from the peak temperature map of ${ }^{13} \mathrm{CO}$ $J=6 \rightarrow 5$, the total column density (Fig. 10b) of ${ }^{13} \mathrm{CO}$ can be calculated over the complete velocity range of the source from

$$
\begin{gathered}
N\left({ }^{13} \mathrm{CO}\right)=1.06 \times 10^{12}\left(T_{\mathrm{ex}}+0.88\right) \exp \left(\frac{116.2}{T_{\mathrm{ex}}}\right) \\
\int T_{\mathrm{MB}}\left({ }^{13} \mathrm{CO}\right) \mathrm{d} v \mathrm{~cm}^{-2},
\end{gathered}
$$

where $T_{\mathrm{ex}}$ is in $\mathrm{K}$ and $T_{\mathrm{MB}} \mathrm{dv}$ is in $\mathrm{K} \mathrm{km} \mathrm{s}^{-1}$. Figure $10 \mathrm{~b}$ shows the resulting ${ }^{13} \mathrm{CO}$ total column density with a peak value of $\sim 5 \times 10^{16} \mathrm{~cm}^{-2}$ northwest of Her 36 . This results in a $\mathrm{H}_{2}$ column 
Table 3. Physical parameters calculated from rotational diagrams.

\begin{tabular}{cccc}
\hline \hline \multicolumn{2}{c}{ Optically thin ${ }^{13} \mathrm{CO}$} & \multicolumn{2}{c}{ Correction factor included } \\
\hline$T_{\text {rot }}^{a}(\mathrm{~K})$ & $N\left({ }^{13} \mathrm{CO}\right)\left(\mathrm{cm}^{-2}\right)$ & $T_{\text {rot }}^{a}(\mathrm{~K})$ & $N\left({ }^{13} \mathrm{CO}\right)\left(\mathrm{cm}^{-2}\right)$ \\
\hline $8.3 \pm 1.3$ & $3.6 \times 10^{16}$ & $8.6 \pm 1.5$ & $6.2 \times 10^{16}$ \\
$41 \pm 3.7$ & $3.6 \times 10^{16}$ & $30 \pm 4.8$ & $5.4 \times 10^{16}$ \\
$90 \pm 21.6$ & $1.9 \times 10^{16}$ & $39 \pm 4.9$ & $4.6 \times 10^{16}$ \\
& & $84 \pm 10$ & $2.1 \times 10^{16}$ \\
\hline Transition & $\tau$ & Transition & $\tau$ \\
\hline$J=1 \rightarrow 0$ & 1.68 & $J=1 \rightarrow 0$ & 1.92 \\
$J=2 \rightarrow 1$ & 1.78 & $J=2 \rightarrow 1$ & 2.20 \\
$J=4 \rightarrow 3$ & 1.25 & $J=4 \rightarrow 3$ & 0.74 \\
$J=6 \rightarrow 5$ & 1.12 & $J=6 \rightarrow 5$ & 0.28 \\
$J=8 \rightarrow 7$ & 1.04 & $J=8 \rightarrow 7$ & 0.10 \\
\hline
\end{tabular}

Notes. ${ }^{(a)}$ Calculated for different gradients with their errors in the plot as indicated in Fig. 11.

density $N\left(\mathrm{H}_{2}\right)$ of $\sim 3.7 \times 10^{22} \mathrm{~cm}^{-2}$ adopting an isotopic abundance ratio $\left[{ }^{12} \mathrm{CO} /{ }^{13} \mathrm{CO}\right]$ of $\sim 63$ (Milam et al. 2005) and a $\mathrm{CO}$ abundance ratio $\left[{ }^{12} \mathrm{CO} / \mathrm{H}_{2}\right]$ of $\sim 8.5 \times 10^{-5}$ (Tielens 2010). The mass of the warm $\mathrm{CO}$ gas can be computed by integrating the column density over the whole clump in a region of $3.12 \mathrm{arcmin}^{2}$, which results in a mass of $\sim 467 M_{\odot}$. Complementary to this, the cold gas mass in a region of $5.1 \mathrm{arcmin}^{2}$ has an estimated value of $10^{3} M_{\odot}$, calculated from a flux of $\sim 133 \mathrm{Jy}$ at $870 \mu \mathrm{m}$ measured with ATLASGAL (Schuller et al. 2009) assuming an absorption coefficient of $k_{v}=1.85 \mathrm{~g} \mathrm{~cm}^{2}$ and a temperature of $23 \mathrm{~K}$ (Urquhart et al. 2018), and not including potential uncertainties in the choice of these values. However, these mass estimations have an error of $\sim 26 \%$, which accounts for errors of $\sim 16 \%$ from the distance to the star (Tothill et al. 2008) and $\sim 20 \%$ from the calibration.

\subsection{Rotational diagrams of ${ }^{13} \mathrm{CO}$}

With observations of $\mathrm{CO}$ lines with different $J$, rotational diagrams can be used to study the excitation of the $\mathrm{CO}$ emitting gas. In a rotational diagram or Boltzmann plot the natural logarithm of the column density $N_{\mathrm{u}} / g_{\mathrm{u}}$ of different lines is plotted against their upper energies $E_{\mathrm{up}} / k$. Here $g_{\mathrm{u}}$ is the degeneracy of the upper energy level, $(\equiv 2 J+1)$, and $k$ is the Boltzmann constant. For a single temperature and optically thin emission these data points fall onto a straight line. Deviations from a straight line indicate then either optical depth effects or temperature gradients in the clouds. A complete derivation of rotational diagrams for a local thermodynamic equilibrium (LTE) case can be found in Goldsmith \& Langer (1999).

Firstly, by assuming ${ }^{13} \mathrm{CO}$ to be optically thin, i.e., the optical depth correction term, $C_{\tau}$ is unity in Eq. (24) of Goldsmith \& Langer (1999), we plot $\ln N_{\mathrm{u}}^{\text {thin }} / g_{\mathrm{u}}$ versus $E_{\mathrm{u}} / K$ as shown in Fig. 11 in black for the five ${ }^{13} \mathrm{CO}$ lines observed toward Her 36. A curvature in a rotational diagram can be due to optical depth effects, therefore we estimate the expected optical depths for the computed column density from Eq. (25) of Goldsmith \& Langer (1999) and apply the optical depth corrections $C_{\tau}$ that lead to the corrected diagram as shown in red in Fig. 11. The new temperatures and column densities are then calculated as shown in Table 3. Further iterations would lead to corrections smaller than the error bars. After the optical depth correction the curvature in the rotational diagram remains

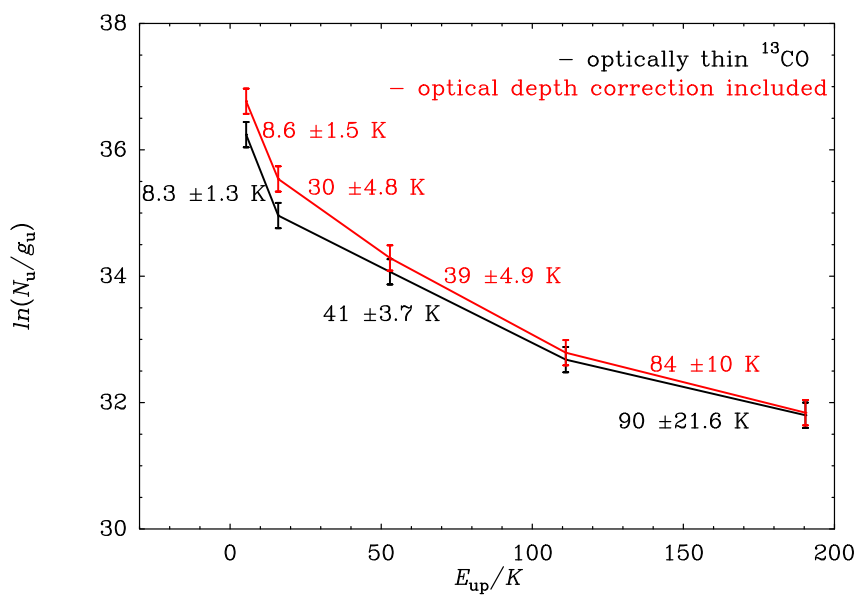

Fig. 11. Rotational diagrams of $J=1 \rightarrow 0, J=2 \rightarrow 1, J=4 \rightarrow 3$, $J=6 \rightarrow 5$, and $J=8 \rightarrow 7$ transitions of ${ }^{13} \mathrm{CO}$ at Her 36. Shown in black is the rotational diagram when ${ }^{13} \mathrm{CO}$ is assumed to be optically thin. In red the rotational diagram is shown including the optical depth correction factors. Rotation temperatures obtained from different slopes are indicated. Values of the velocity integrated intensities for different transitions were extracted from maps convolved to the same resolution of $31^{\prime \prime}$. The error bars were calculated from the maximum noise level of the integrated intensities of individual transitions and from calibration uncertainties of $20 \%$.

and indicates temperature gradients in the gas, as expected in a PDR.

The $J=1 \rightarrow 0$ and $J=2 \rightarrow 1$ transitions of ${ }^{13} \mathrm{CO}$ seem to originate from colder gas, while the $J=4 \rightarrow 3$ and $J=6 \rightarrow 5$ transitions probe hotter gas, which agrees with the analysis carried out for $J=6 \rightarrow 5$ in Sect. 4.1. The $J=8 \rightarrow 7$ transition appears to originate from the hottest gas.

\subsection{RADEX modeling}

We used the non-LTE radiative transfer program RADEX (van der Tak et al. 2007) to verify the calculations carried out in Sects. 4.1 and 4.2, which assumed LTE to determine the temperatures and densities. The RADEX program uses the escape probability approximation for a homogeneous medium and takes into account optical depth effects. We chose a uniform sphere geometry. The Leiden Molecular and Atomic Database ${ }^{8}$ (LAMDA; Schöier et al. 2005) provides rates coefficients for collisions of $\mathrm{CO}$ and $\mathrm{H}_{2}$ used in the modeling. ${ }^{12} \mathrm{CO}$ and ${ }^{13} \mathrm{CO}$ transitions were modeled taking their line width from the average spectra of our data, i.e., 4 and $3 \mathrm{~km} \mathrm{~s}^{-1}$, respectively. As input parameters we computed grids in temperature and volume density with a background temperature of $2.73 \mathrm{~K}$, kinetic temperatures in the range of $50-250 \mathrm{~K}$ and $\mathrm{H}_{2}$ densities in the range of $10^{4}-10^{8} \mathrm{~cm}^{-3}$. For a linear molecule line ratios from different $J$ depend on both temperature and density. To break this degeneracy, we computed with RADEX not only the line ratio of two ${ }^{13} \mathrm{CO}$ transitions but also the temperature of the ${ }^{12} \mathrm{CO}$ line. The latter is optically thick, probes the excitation temperature (cf. Sect. 4.1), and can therefore be used to break the degeneracy between temperature and density.

In our first approach to determine the dominant kinetic temperatures and densities in M8 near Her 36, we selected from the $\mathrm{CO}$ maps data points for column densities of ${ }^{12} \mathrm{CO}$ in three ranges as follows: (a) $8 \times 10^{17}-1.8 \times 10^{18} \mathrm{~cm}^{-2}$, (b) $1.8 \times 10^{18}$ $3.5 \times 10^{18} \mathrm{~cm}^{-2}$, and (c) $3.5 \times 10^{18}-5.1 \times 10^{18} \mathrm{~cm}^{-2}$. These

8 http://www.strw.leidenuniv.nl/ moldata/ 

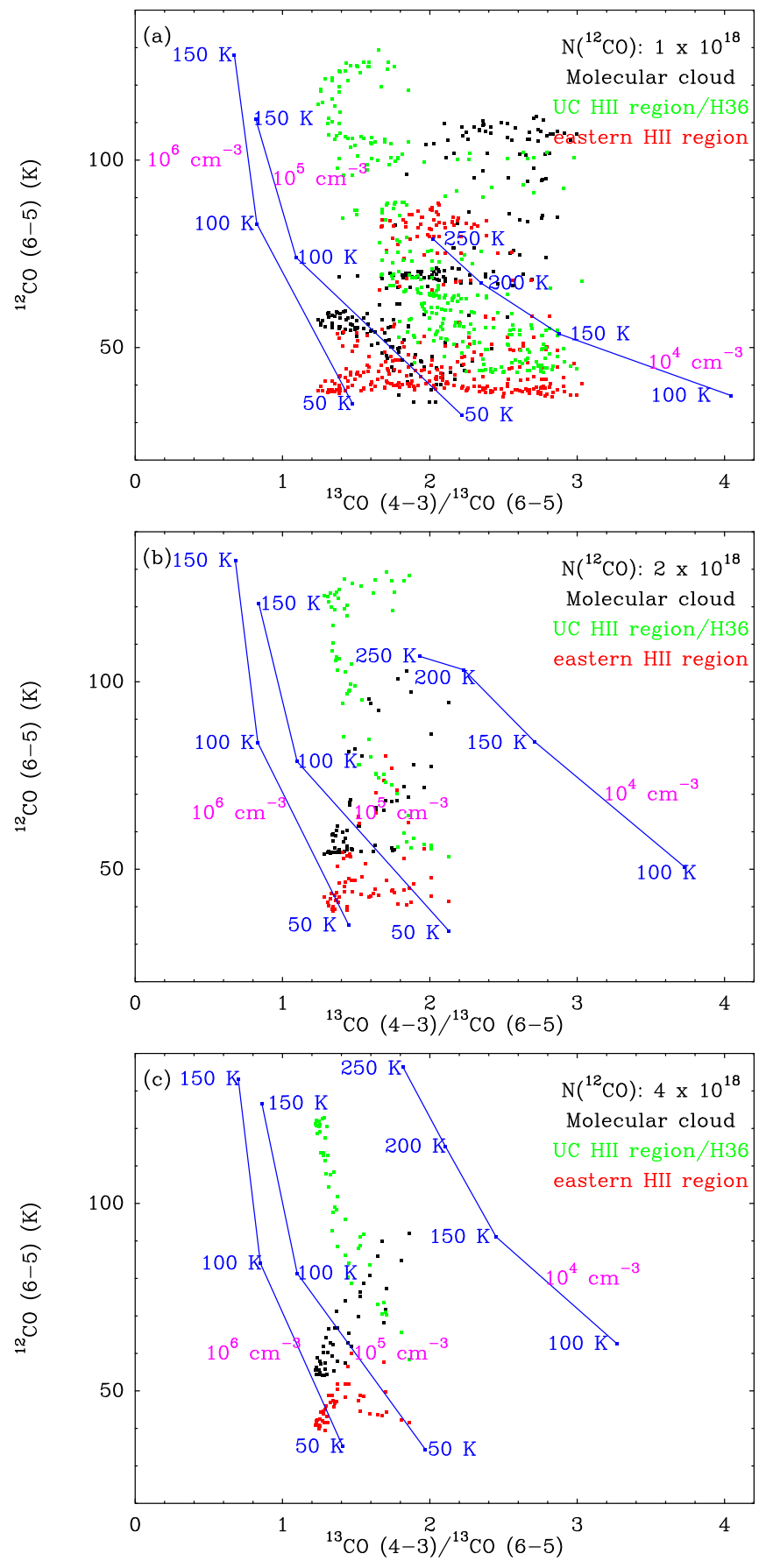

Fig. 12. For 3 different ${ }^{12} \mathrm{CO}$ column densities, RADEX modeling results are shown for ${ }^{12} \mathrm{CO} J=6 \rightarrow 5$ peak main-beam brightness temperatures and corresponding ${ }^{13} \mathrm{CO} J=4 \rightarrow 3 /{ }^{13} \mathrm{CO} J=6 \rightarrow 5$ ratios. The blue lines denote the RADEX modeling results for different kinetic temperatures at 3 different volume densities, and color points represent data from near the HII region, PDR, and molecular cloud (see Sect. 4.3). Each point denotes a ${ }^{12} \mathrm{CO} J=6 \rightarrow 5$ temperature and ${ }^{13} \mathrm{CO} J=4 \rightarrow 3 /{ }^{13} \mathrm{CO} J=6 \rightarrow 5$ temperature ratio obtained from a single pixel. Data points are extracted from peak temperature maps convolved to the same resolution of $16^{\prime \prime}$. (a) The data points plotted are obtained for a ${ }^{12} \mathrm{CO}$ column density range of $8 \times 10^{17}-1.8 \times 10^{18} \mathrm{~cm}^{-2}$ and for modeling the chosen input ${ }^{12} \mathrm{CO}$ column density is $1 \times 10^{18} \mathrm{~cm}^{-2}$. (b) The data points plotted are obtained for a ${ }^{12} \mathrm{CO}$ column density range of $1.8 \times 10^{18}-3.5 \times$ $10^{18} \mathrm{~cm}^{-2}$ and for the modeling the input ${ }^{12} \mathrm{CO}$ column density is $2 \times 10^{18} \mathrm{~cm}^{-2}$. (c) The data points plotted are obtained for a ${ }^{12} \mathrm{CO}$ column density range of $3.5 \times 10^{18}-5.1 \times 10^{18} \mathrm{~cm}^{-2}$ and for the modeling input the ${ }^{12} \mathrm{CO}$ column density is $4 \times 10^{18} \mathrm{~cm}^{-2}$. ranges were estimated from the LTE calculations carried out in Sects. 4.1 and 4.2. Another criterium for selection of these data points aims at selecting the most conspicuous regions inside the maps. The molecular cloud region consists of all data points in an area of $15^{\prime \prime} \times 15^{\prime \prime}$ around the mid- $J$ CO peak, the UC HII region/ Her 36 encompasses all data points in an area of $20^{\prime \prime} \times 20^{\prime \prime}$ around Her 36, and the eastern HII region has data points in an area of $20^{\prime \prime} \times 20^{\prime \prime}$ around the $[\mathrm{C} \mathrm{II}]$ peak.

Figure 12 shows the $J=4 \rightarrow 3$ and $J=6 \rightarrow 5$ line ratios of ${ }^{13} \mathrm{CO}$ vs. the $J=6 \rightarrow 5{ }^{12} \mathrm{CO}$ line temperature for both the measured data points in the maps and the results of the RADEX computation of the density/temperature grid. The $\mathrm{H}_{2}$ number densities in most of the regions are in the range of $\sim 10^{4}-10^{6} \mathrm{~cm}^{-3}$. High densities are obtained for the molecular cloud and the eastern HII region, while the region consisting of the bright stellar system Her 36 and the ultracompact HII region has densities in the range of $10^{4}-10^{5} \mathrm{~cm}^{-3}$. The kinetic temperatures from the modeling results are in a range of 100-250 K for $10^{4} \mathrm{~cm}^{-3}$ and in a range of $50-150 \mathrm{~K}$ for $10^{5}-10^{6} \mathrm{~cm}^{-3}$.

In order to include all $\mathrm{CO}$ lines toward several positions in the RADEX analysis, their intensities as a function of $J$ are compared to RADEX results in Fig. 13. To fit the modeling results to the observed data points we varied the column densities of ${ }^{12} \mathrm{CO}$ and ${ }^{13} \mathrm{CO}$. While for ${ }^{12} \mathrm{CO}$ a column density of $4 \times 10^{18} \mathrm{~cm}^{-2}$ was chosen, for ${ }^{13} \mathrm{CO}$ a column density of $8 \times 10^{16} \mathrm{~cm}^{-2}$ could make the modeling results fit the data points. This ${ }^{13} \mathrm{CO}$ column density exceeds the value obtained by the LTE calculations and corresponds to a ${ }^{12} \mathrm{CO} /{ }^{13} \mathrm{CO}$ ratio of 50 . While this is lower than the assumed value of 63 in Sect. 4.1, it is still within the typical scatter of this ratio in the ISM (Milam et al. 2005). The ${ }^{12} \mathrm{CO}$ and ${ }^{13} \mathrm{CO}$ observed data is compared to the RADEX model at the peak of the mid- $J$ CO transitions in the molecular cloud, at Her 36 and at the emission peak of [C II]. Panels $a$ and $b$ of Fig. 13 show results with the kinetic temperature varied from $50-250 \mathrm{~K}$ and keeping the $\mathrm{H}_{2}$ density fixed at $10^{5} \mathrm{~cm}^{-3}$, while panels $\mathrm{c}$ and $\mathrm{d}$ show results in which the $\mathrm{H}_{2}$ density is varied from $10^{4}-10^{8} \mathrm{~cm}^{-3}$ while keeping the kinetic temperature fixed at $120 \mathrm{~K}$.

It can be seen that no single kinetic temperature or $\mathrm{H}_{2}$ density can fit all the observed data points. This suggests solutions with kinetic temperatures in the range of $100-150 \mathrm{~K}$ and $\mathrm{H}_{2}$ densities to be in the range of $10^{4}-10^{6} \mathrm{~cm}^{-3}$. Such a spread in the ambient temperature was also implied by the rotational diagram analysis. Furthermore, these values are similar to the temperature and density ranges found in OMC 1 (Peng et al. 2012).

\section{4. $\mathrm{CO},[\mathrm{C} \mathrm{I}]$ and $[\mathrm{C} \mathrm{II}]$ luminosities}

We obtained the total luminosities of the $\mathrm{CO}$ spectral line energy distributions (SLED) and of the [C I] $609 \mu \mathrm{m}$ and [C II] lines over the total observed region seen in the maps in Figs. 2-4, as derived by Solomon et al. (1997), Carilli \& Walter (2013). We scaled the luminosity for Galactic sources, i.e.,

$$
L=1.04 \times 10^{-9} S \Delta V v D_{\mathrm{L}}^{2},
$$

where $L$ is the line luminosity in $L_{\odot}, S \Delta V$ is the velocity integrated flux in $\mathrm{Jy} \mathrm{km} \mathrm{s} \mathrm{km}^{-1}, v$ is the transition frequency in $\mathrm{GHz}$, and $D_{\mathrm{L}}$ is the distance in kpc. A total CO luminosity of $L_{\mathrm{CO}}=$ 9.5 $L_{\odot}$ was calculated for the observed transitions and by accounting for the luminosities of the missing transitions. A [C I] $609 \mu \mathrm{m}$ line luminosity of $L_{\left[\mathrm{C}_{\mathrm{I}}\right]}=0.11 L_{\odot}$ was obtained, which is a lower limit to the total [ $\left.\mathrm{C}_{\mathrm{I}}\right]$ luminosity since the $\left[\mathrm{C}_{\mathrm{I}}\right]$ $370 \mu \mathrm{m}$ line was not observed. The estimated [C II] luminosity is 

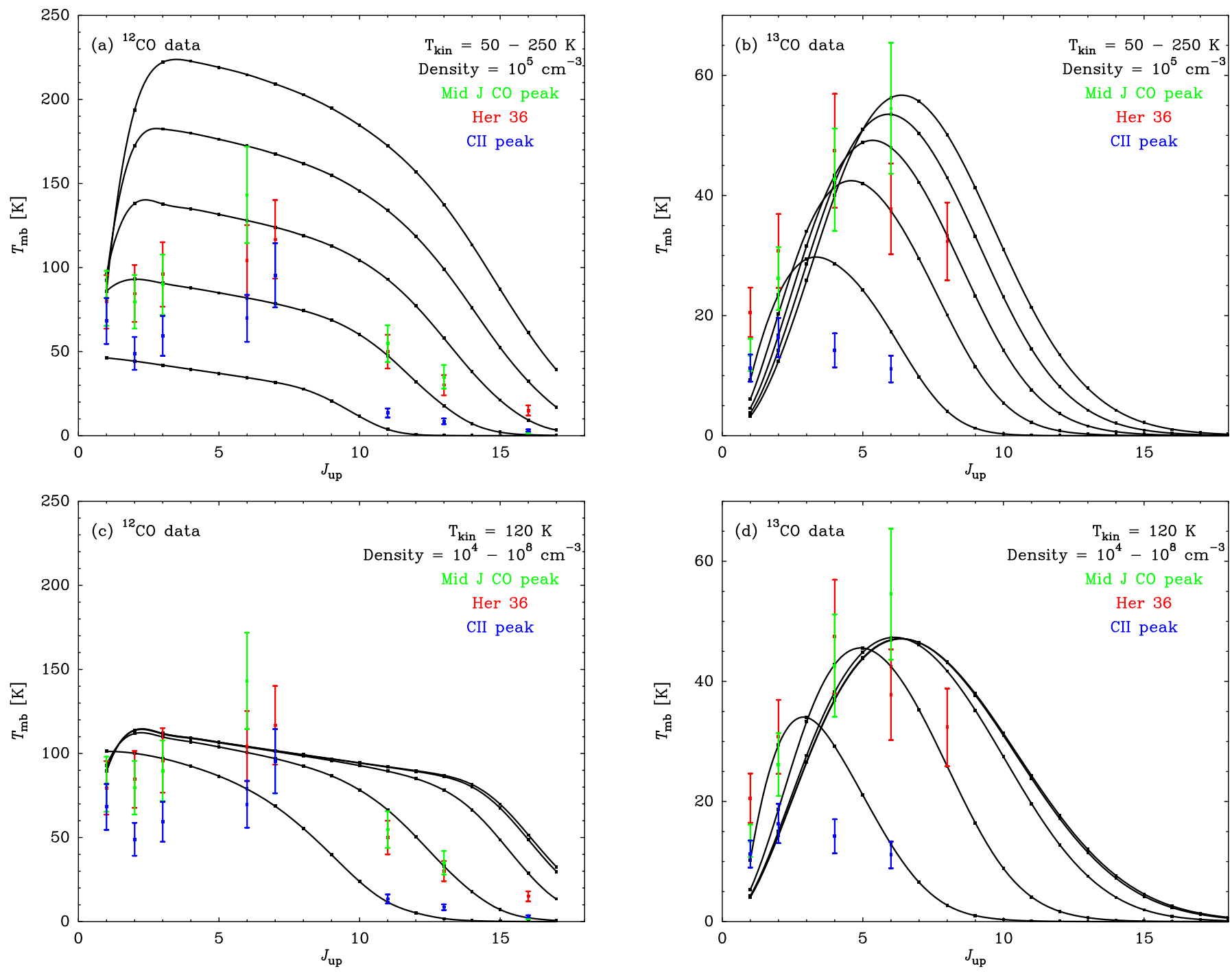

Fig. 13. Results obtained from RADEX modeling for ${ }^{12} \mathrm{CO}$ and ${ }^{13} \mathrm{CO}$ at the mid- $J$ CO peak $\left(\Delta \alpha=-13.0^{\prime \prime}, \Delta \delta=8.0^{\prime \prime}\right)$, at $\mathrm{Her} 36\left(\Delta \alpha=00^{\prime \prime}\right.$, $\left.\Delta \delta=0^{\prime \prime}\right)$ and at the $\left[\mathrm{C}\right.$ II] peak $\left(\Delta \alpha=30.0^{\prime \prime}, \Delta \delta=-2.0^{\prime \prime}\right)$. Our observed data points are in green, red, and blue and are extracted from peak temperature maps convolved to the same resolution of $31^{\prime \prime}$. The column densities used in the modeling are for ${ }^{12} \mathrm{CO} 4 \times 10^{18} \mathrm{~cm}^{-2}$ and $8 \times 10^{16}$ $\mathrm{cm}^{-2}$ for ${ }^{13} \mathrm{CO}$. Panels $a$ and $b$ : results obtained by varying the kinetic temperature from $50-250 \mathrm{~K}$ in steps of $50 \mathrm{~K}$ and keeping the density fixed at $10^{5} \mathrm{~cm}^{-3}$; panels $c$ and $d$ : results obtained by varying the $\mathrm{H}_{2}$ density from $10^{4}-10^{8} \mathrm{~cm}^{-3}$ in steps by a factor of 10 and keeping the kinetic temperature fixed at $120 \mathrm{~K}$.

$L_{\left[\mathrm{C}_{\text {II }}\right]}=95.8 L_{\odot}$. Similar to the mass estimations in Sect. 4.1, these luminosity estimations have an error of $\sim 26 \%$.

\section{Discussion}

\subsection{Overview of the PDR and HII region around Her 36}

In Fig. 14, we present a F487N filtered $4865 \AA$ HST image 9 (observation ID number: 6227, observed in the year 1995) of Her 36 and its surroundings overlaid with contours from low velocity $[\mathrm{C}$ II] channel maps. The $[\mathrm{C}$ II] at the lowest velocity $\left(2 \mathrm{~km} \mathrm{~s}^{-1}\right)$ peaks at the Hourglass Nebula slightly to the east of

9 Based on observations made with NASA/ESA Hubble Space Telescope, and obtained from the Hubble Legacy Archive, which is a collaboration between the Space Telescope Science Institute (STScI/NASA), the Space Telescope European Coordinating Facility (ST-ECF/ESA) and the Canadian Astronomy Data Centre (CADC/NRC/CSA).
Her 36. With increasing velocities, the [C II] follows the dark patches in the HST images that form a foreground veil covering parts of the bright nebulosity excited by Her 36 . The strong correlation of $[\mathrm{C} \mathrm{II}]$ and foreground absorption is particularly evident at the sharp southern edge of the veil seen at $7 \mathrm{~km}$ $\mathrm{s}^{-1}$. Therefore we suggest that the low velocity [C II] probes directly the gas of the veil that forms a foreground PDR illuminated by Her 36. On a fainter level, weak emission from this veil is also seen in the $\mathrm{CO}$ maps at low velocities $(5-7 \mathrm{~km}$ $\left.\mathrm{s}^{-1}\right)$. This foreground veil is receding away from Her 36 toward us and to the west with a change in the line-of-sight velocity. This is consistent with both high velocity red-shifted and low velocity blue-shifted emission of $\mathrm{H} \alpha,[\mathrm{N}$ II] and [S II] doublets, [O III], and absorption lines of the sodium D doublet as measured by Damiani et al. (2017). Assuming optically thin emission from $[\mathrm{C} \mathrm{II}]$ in this warm veil in the velocity range of $2-7 \mathrm{~km}$ $\mathrm{s}^{-1}$ and a kinetic temperature of $500 \mathrm{~K}$ (Tielens \& Hollenbach 1985b), we calculated the [C II] column density using Eq. (A.1) with a peak value of $\sim 9.6 \times 10^{17} \mathrm{~cm}^{-2}$ at an offset of $\left(\Delta \alpha=30^{\prime \prime}\right.$, 

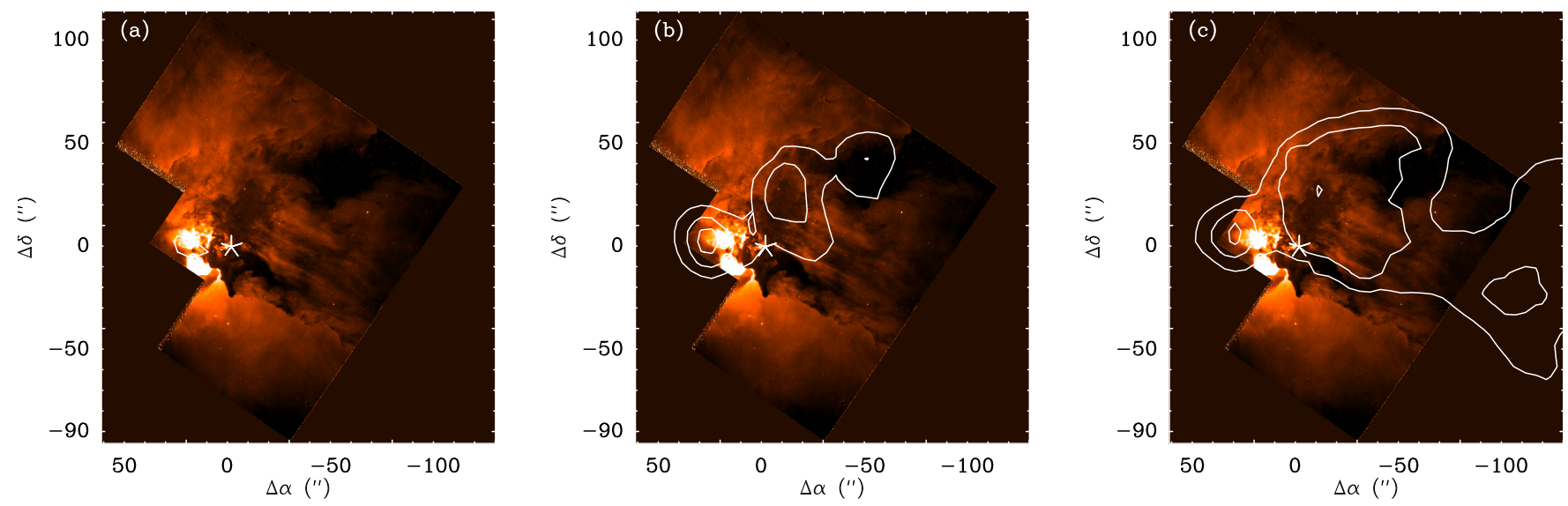

Fig. 14. HST F487N (4865 $\AA$ ) image of Her 36 and its surroundings overlaid with [C II] channel map contours (in white) at $(a) 2 \mathrm{~km} \mathrm{~s}^{-1},(b)$ $5 \mathrm{~km} \mathrm{~s}^{-1}$, and $(c) 7 \mathrm{~km} \mathrm{~s}^{-1}$ to show the progression of the foreground material at lower velocities. Her 36 is the central position $(\Delta \alpha=0, \Delta \delta=0)$ at $\mathrm{RA}(\mathrm{J} 2000)$ denoted with an asterisk at $\mathrm{RA}(\mathrm{J} 2000)=18^{\mathrm{h}} 03^{\mathrm{m}} 40.3^{\mathrm{s}}$ and $\operatorname{Dec}(\mathrm{J} 2000)=-24^{\circ} 22^{\prime} 43^{\prime \prime}$, with the hourglass nebula representing the two bright hotspots about $\sim 15^{\prime \prime}$ to the east. Contour levels are from $10 \%$ to $100 \%$ in steps of $10 \%$ of the peak emission observed at the channel with $10 \mathrm{~km} \mathrm{~s}^{-1}$ velocity.
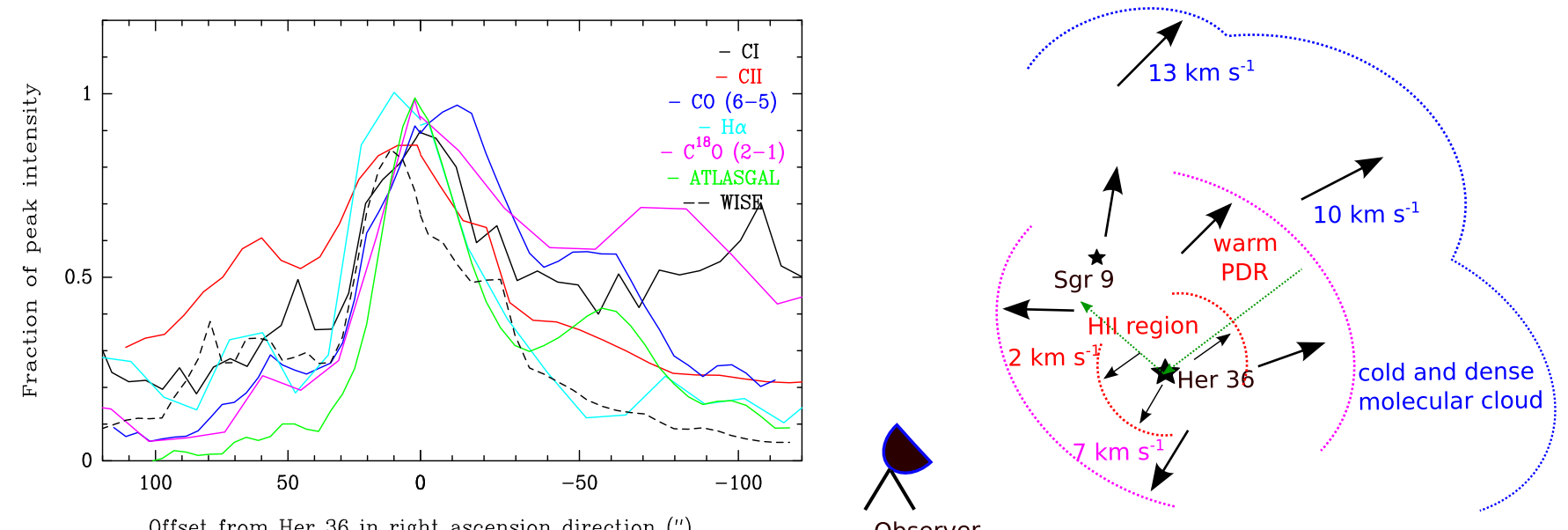

Fig. 15. Left panel: velocity integrated intensities normalized to their value at peak vs. offset (") from Her 36 along the green arrows shown in the right. They follow a path from the second ATLASGAL peak at $\left(-53^{\prime \prime}, 23^{\prime \prime}\right)$ to the second [C II] peak at $\left(60^{\prime \prime}, 27^{\prime \prime}\right)$ via Her 36 at $\left(0^{\prime \prime}, 0^{\prime \prime}\right)$. Plots are shown for [C II], [C I], $J=6 \rightarrow 5{ }^{12} \mathrm{CO}, J=2 \rightarrow 1 \mathrm{C}^{18} \mathrm{O}, \mathrm{H} \alpha$, ATLASGAL $870 \mu \mathrm{m}$, and WISE $3.4 \mu \mathrm{m}$ emission; right panel: schematic diagram of the prominent optical features of M8 pertinent to the discussion in this paper. The cold and dense molecular cloud is in the background shown in blue. The foreground gas of the warm PDR veil is receding away from Her $36\left(\sim 9 \mathrm{~km} \mathrm{~s}^{-1}\right)$ with lower velocities $\left(2-7 \mathrm{~km} \mathrm{~s}{ }^{-1}\right)$, while the HII region is powered by both stellar systems, Her 36 and $9 \mathrm{Sgr}$.

$\Delta \delta=10^{\prime \prime}$ ) from Her 36. Taking $\mathrm{C} / \mathrm{H} \sim 1.2 \times 10^{-4}$, assuming that all the carbon is in ionized form and from the column density of $\mathrm{H}_{2}, N\left(\mathrm{H}_{2}\right)=9.4 \times 10^{20} \mathrm{~cm}^{-2}\left(A_{\mathrm{v}} / \mathrm{mag}\right.$ ) (Kauffmann et al. 2008), we derived the visual extinction $A_{\mathrm{v}}$. A maximum extinction of $A_{\mathrm{V}} \sim 4.25$ is obtained at the same position where the [C II] column density peaks and the values get lower around it. This position is the same as position 13 in Fig. 5 of Woodward et al. (1986), where an $A_{\mathrm{v}} \sim 3.9$ was calculated.

In Fig. 15 (left) we show how the intensity of various tracers evolves along a path from the direction of 9 Sgr to Her 36 and then continuing to the northwest along the molecular cloud. Toward the northeast [C II] and the mid-IR WISE emission dominate, probing the extended HII regions toward $9 \mathrm{Sgr}$ and the resulting PDR. All of the tracers peak on or close to Her 36, showing the tight spatial correlation between the ultracompact HII region (as seen in the recombination line), a dense clump in the larger scale molecular cloud $(870 \mu \mathrm{m}$ dust and molecular emission), and the bright PDR illuminated by Her 36 ([C II] and WISE). To the northwest, emission from the molecular cloud dominates with the second dense but colder clump at an offset of about $70^{\prime \prime}$ from Her 36. [C $\mathrm{I}$ ] diffuse emission is very extended in this region.

Taking this into consideration along with our analysis of the morphology carried out in Sects. 3 and 4, we propose a geometry of the region illuminated by Her 36 and 9 Sgr as presented in Fig. 15 (right). We propose that the cold dense molecular cloud is behind the bright stars Her 36 and 9 Sgr. Her 36 is still much closer to the dense part of the cloud in which it was born; in fact the foreground veil is part of the original cloud accelerated toward us by the strong radiation and wind of Her 36, showing the expansion of the nebula, blue shifted with respect to the molecular cloud (3-7 $\mathrm{km} \mathrm{s}^{-1}$ ) moving away from Her 36 $\left(\sim 9 \mathrm{~km} \mathrm{~s}^{-1}\right)$ toward the observer and the west, while the red-shifted (11-13 $\mathrm{km} \mathrm{s}^{-1}$ ) [C II] probes the background material toward the northeast of Her 36. The ultracompact HII region is fueled by Her 36 around its vicinity and the more extended diffuse HII region by $9 \mathrm{Sgr}$ toward the east (Tothill et al. 2008). 
Table 4. CO $J=2 \rightarrow 1$, [C II] and FIR luminosity ratios of M8 and the Orion Bar.

\begin{tabular}{ccc}
\hline \hline Luminosity ratio & $\mathrm{M} 8$ & Orion Bar \\
\hline$L_{[\mathrm{C} \text { II }} / L_{\mathrm{FIR}}$ & $10^{-3}$ & $1.1 \times 10^{-3}$ \\
$L_{\left[\mathrm{C}_{\mathrm{II}}\right]} / L_{\mathrm{CO} 2-1}$ & $7.5 \times 10^{2}$ & $1.6 \times 10^{3}$ \\
$L_{\mathrm{CO} 2-1} / L_{\mathrm{FIR}}$ & $1.3 \times 10^{-6}$ & $6.6 \times 10^{-7}$ \\
\hline
\end{tabular}

\subsection{Comparison with PDR of the Orion Bar}

The Orion Bar is a well-known PDR and its properties are described in depth by Hollenbach \& Tielens (1997) and Walmsley et al. (2000). It is part of the OMC-1 core at the edge of M42 and is illuminated by young massive stars, which form a trapezium at the center of the Orion Nebular Cluster, mainly from $\theta^{1} \mathrm{C}$, which is a O5-O7 star. Also, the PDR appears to be located at the edge of the HII blister tangential to the line of sight (Peng et al. 2012). A comparison of $L_{\mathrm{CO} 2-1}, L_{[\mathrm{C} \text { II }]}$ and $L_{\text {FIR }}$ between M8 and the Orion Bar is presented in Table 4. For M8, the $L_{\mathrm{CO} 2-1}=0.128 L_{\odot}$ (calculated in a similar way as in Sect. 4.4), $L_{\left[\mathrm{C}_{\mathrm{II}}\right]}$ is calculated in Sect. 4.4 and $L_{\mathrm{FIR}} \sim 10^{4} L_{\odot}$ is obtained from White et al. (1998). The luminosities of the Orion Bar are taken from Goicoechea et al. (2015).

We calculated the FUV radiation field, $G_{0} \sim 0.6-1.12 \times 10^{5}$ in Habing units and density, $n \sim 0.97-1.93 \times 10^{5} \mathrm{~cm}^{-3}$ for the PDR of M8 by adopting an electron density $n_{\mathrm{e}}$ of 2000-4000 $\mathrm{cm}^{-3}$, electron temperature $T_{\mathrm{e}}$ of $7000-9000 \mathrm{~K}$ (Woodward et al. 1986; Esteban et al. 1999). We used the values of stellar luminosity and number of ionizing photons for an O7 star from Sect. 7.2.1 of Tielens (2010). The densities match well with the RADEX calculations carried out in Sect. 4.4. Interestingly, these calculated values of $G_{0}$ and $n$ also match very well with those calculated for the Orion Bar. This leads us to a direct comparison of the results of the PDR models of the Orion Bar (Tielens \& Hollenbach 1985a,b; Jansen et al. 1995; Hogerheijde et al. 1995; Hollenbach \& Tielens 1997, 1999; Andree-Labsch et al. 2017) with the PDR of M8 since we expect similar chemical and thermal conditions in both PDRs. Tielens \& Hollenbach (1985a) and Hollenbach \& Tielens (1999) calculated the structure of the Orion Bar as a function of visual extinction $A_{\mathrm{v}}$. They show a typical case in which $\mathrm{H}_{2}$ does not self-shield until dust attenuation of the FUV photons creates an atomic surface layer. According to this, in the PDR of M8 the transition of atomic $\mathrm{H}$ to molecular $\mathrm{H}_{2}$ occurs at $A_{\mathrm{v}}=2$, the carbon balance shifts from $\mathrm{C}^{+}$to $\mathrm{C}$ and $\mathrm{CO}$ at $A_{\mathrm{v}}=4$, and except for the $\mathrm{O}$ in $\mathrm{CO}$, all oxygen is in atomic form until very deep into the molecular cloud at $A_{\mathrm{v}}=8$. The gas in the surface layer is much warmer at about $\sim 500 \mathrm{~K}$ than the dust, which is at about $\sim 30-75 \mathrm{~K}$. Complementary to the $\mathrm{H}_{2}$ column density calculation carried out in Sect. 4.1, assuming a dust temperature of $75 \mathrm{~K}$ and a maximum flux value of $5000 \mathrm{mJy} \mathrm{beam}^{-1}$ obtained from ATLASGAL data, allowed us to calculate the $\mathrm{H}_{2}$ column density at Her $36, N\left(\mathrm{H}_{2}\right) \sim 3.75 \times 10^{22} \mathrm{~cm}^{-2}$ which is in reasonable agreement with that calculated in Sect. 4.1 within a factor $\sim 1.25$.

Hogerheijde et al. (1995), Walmsley et al. (2000), and Andree-Labsch et al. (2017) described the geometry of the Orion Bar where the trapezium stars illuminate the PDR, which changes from a face-on to an edge-on orientation along the varying length of the line of sight. This geometry explains the [C $\mathrm{I}]$ peak that is symmetric around the peak of the $\mathrm{CO}$ emission (Tauber et al. 1994). The [C II] emission peak is also distributed symmetrically around the ionization front (Stacey et al. 1993). Contrary to this, in $\mathrm{M} 8$, we see that [C II] peaks at the east of Her 36, [C I] peaks at Her 36, while the CO transitions peak in the northwest of Her 36, which supports the proposition of a face-on geometry.

\subsection{Comparison with the PDR of M17 SW}

M17, the Omega nebula is also among the best nearby laboratories to study star formation. It has an edge-on geometry in contrast to the face-on geometry of M8. It has a bright HII region ionized by the rich cluster NGC 6618 (Povich et al. 2009) and beyond this HII region lies the bright PDR in the southwest of M17 (M17 SW), which is responsible for the photoelectric heating of the warm gas (Pérez-Beaupuits et al. 2015a). M17 SW also contains a wide ranged clumpy molecular cloud studied widely by Pérez-Beaupuits et al. (2010) and Pérez-Beaupuits et al. (2015a).

In Sect. 3.2, the scatter plots related to M8 show only a weak correlation of $\left[\mathrm{C}_{\mathrm{II}}\right]$ with $\left[\mathrm{C}_{\mathrm{I}}\right]$ and ${ }^{12} \mathrm{CO}$. The channel maps and line profiles at different offsets also show different morphologies of [C II] compared with those seen in [C I $]$ and $\mathrm{CO}$ except in a small range of intermediate velocities toward Her 36. This suggests that $[\mathrm{C}$ II] and the molecular gas tracers on scales away from Her 36 do not originate from the same spatial region. This is similar to M17 SW as reported by Pérez-Beaupuits et al. (2015a). In Sect. 4.3, the comparison between the observed ${ }^{12} \mathrm{CO}$ and ${ }^{13} \mathrm{CO}$ data with non-LTE RADEX modeling results show that the UC HII region or molecular gas near the Her 36 region has the highest density and kinetic temperature, while the molecular gas near the eastern HII region has low density and lower kinetic temperature. In contrast to M17 SW (Pérez-Beaupuits et al. 2015b), the ${ }^{12}$ CO SLED shapes we see in Fig. 13 toward Her 36 and mid- $J$ CO positions follow a similar trend. Thus, they do not indicate large fluctuations in gas temperatures of the molecular gas. However, similarly to the case of M17 SW, the higher $J$ CO lines show significantly lower intensities at the [C II] peak position. This is consistent with a PDR, where the [C II] peak emission is expected to arise from less dense gas than at the Her 36 position.

\section{Conclusions}

In this paper, we presented for the first time velocity integrated intensity maps of $J=11 \rightarrow 10, J=13 \rightarrow 12, J=16 \rightarrow 15^{12} \mathrm{CO}$, and [C II] $158 \mu \mathrm{m}$, observed toward Her 36 in M8 using the dualcolor Terahertz receiver GREAT on board the SOFIA telescope; $J=2 \rightarrow 1, J=3 \rightarrow 2, J=6 \rightarrow 5$, and $J=7 \rightarrow 6{ }^{12} \mathrm{CO}$ transitions; $J=2 \rightarrow 1, J=4 \rightarrow 3$, and $J=6 \rightarrow 5{ }^{13} \mathrm{CO}$ transitions using the $\mathrm{CHAMP}^{+}, \mathrm{FLASH}^{+}$, and PI230 receivers of the APEX telescope; and $J=1 \rightarrow 0$ transitions of ${ }^{12} \mathrm{CO}$ and ${ }^{13} \mathrm{CO}$ using the EMIR receiver of IRAM 30 m telescope.

Combining the information obtained from Sects. 3 and 5.1, we put forward the geometry of the region surrounding Her 36. M8 has a face-on geometry where the cold dense molecular cloud lies in the background with Her 36 being still very close to the dense core of the cloud from which it was born. Her 36 is powering the HII region toward the east of it along with 9 Sgr, while the foreground veil of a warm PDR is receding away (at lower velocities) from Her 36 toward the observer.

Using different techniques we studied the physical conditions in the molecular gas associated with M8. CO rotation diagrams indicate temperature gradients through the PDR. Low- $J$ 
${ }^{13} \mathrm{CO}$ transitions seem to originate from colder gas, while the $J=8 \rightarrow 7$ transition seems to originate from the hottest gas. Quantitative analysis including LTE approximation methods and the non-LTE RADEX program were used to calculate the temperatures and $\mathrm{H}_{2}$ number density in the PDR around Her 36. Kinetic temperatures ranging from $100-150 \mathrm{~K}$ and densities ranging from $10^{4}-10^{6} \mathrm{~cm}^{-3}$ were obtained.

Acknowledgements. SOFIA is jointly operated by the Universities Space Research Association, Inc. (USRA), under NASA contract NAS2-97001, and the Deutsches SOFIA Institut (DSI) under DLR contract 50 OK 0901 and 50 OK 1301 to the University of Stuttgart. We are thankful to the SOFIA operations team for their help and support during and after the observations. M. Tiwari was supported for this research by the International Max-Planck-Research School (IMPRS) for Astronomy and Astrophysics at the Universities of Bonn and Cologne. We also thank Thushara Pillai for helpful discussions. The research reported here was motivated by a discussion between Don York and Karl Menten.

\section{References}

Andree-Labsch, S., Ossenkopf-Okada, V., \& Röllig, M. 2017, A\&A, 598, A2 Arias, J. I., Barbá, R. H., Gamen, R. C., et al. 2010, ApJ, 710, L30

Arias, J. I., Barbá, R. H., Maíz Apellániz, J., Morrell, N. I., \& Rubio, M. 2006, MNRAS, 366, 739

Burton, M. G. 2002, PASA, 19, 260

Carilli, C. L., \& Walter, F. 2013, ARA\&A, 51, 105

Carter, M., Lazareff, B., Maier, D., et al. 2012, A\&A, 538, A89

Chen, L., de Grijs, R., \& Zhao, J. L. 2007, AJ, 134, 1368

Dahlstrom, J., York, D. G., Welty, D. E., et al. 2013, ApJ, 773, 41

Damiani, F., Bonito, R., Prisinzano, L., et al. 2017, A\&A, 604, A135

Damiani, F., Flaccomio, E., Micela, G., et al. 2004, ApJ, 608, 78

Draine, B. T. 2011, Physics of the Interstellar and Intergalactic Medium (Princeton, Oxford: Princeton University Press)

Esteban, C., Peimbert, M., Torres-Peimbert, S., García-Rojas, J., \& Rodríguez, M. 1999, ApJS, 120, 113

Goicoechea, J. R., Teyssier, D., Etxaluze, M., et al. 2015, ApJ, 812, 75

Goldsmith, P. F., \& Langer, W. D. 1999, ApJ, 517, 209

Goto, M., Stecklum, B., Linz, H., et al. 2006, ApJ, 649, 299

Guan, X., Stutzki, J., Graf, U. U., et al. 2012, A\&A, 542, L4

Güsten, R., Booth, R. S., Cesarsky, C., et al. 2006, SPIE Conf. Ser., 6267, 626714

Heyminck, S., Graf, U. U., Güsten, R., et al. 2012, A\&A, 542, L1

Hogerheijde, M. R., Jansen, D. J., \& van Dishoeck, E. F. 1995, A\&A, 294, 792

Hollenbach, D. J., \& Tielens, A. G. G. M. 1997, ARA\&A, 35, 179

Hollenbach, D. J., \& Tielens, A. G. G. M. 1999, Rev. Mod. Phys., 71, 173

Jansen, D. J., Spaans, M., Hogerheijde, M. R., \& van Dishoeck, E. F. 1995, A\&A, 303,541

Kauffmann, J., Bertoldi, F., Bourke, T. L., Evans, II, N. J., \& Lee, C. W. 2008, A\&A, 487, 993

Klein, B., Hochgürtel, S., Krämer, I., et al. 2012, A\&A, 542, L3

Lada, C. J., Gottlieb, C. A., Gottlieb, E. W., \& Gull, T. R. 1976, ApJ, 203, 159

Lynds, B. T., \& Oneil, Jr. E. J. 1982, ApJ, 263, 130

Mangum, J. G., \& Shirley, Y. L. 2016, PASP, 128, 029201

Masqué, J. M., Dzib, S., \& Rodríguez, L. F. 2014, ApJ, 797, 60

Milam, S. N., Savage, C., Brewster, M. A., Ziurys, L. M., \& Wyckoff, S. 2005, ApJ, 634, 1126

Oka, T., Welty, D. E., Johnson, S., et al. 2014, ApJ, 793, 68
Pabst, C. H. M., Goicoechea, J. R., Teyssier, D., et al. 2017, A\&A, 606, A29 Peng, T.-C., Wyrowski, F., Zapata, L. A., Güsten, R., \& Menten, K. M. 2012 , A\&A, 538, A12

Pérez-Beaupuits, J. P., Güsten, R., Spaans, M., et al. 2015a, EAS Pub. Ser., 75, 205

Pérez-Beaupuits, J. P., Güsten, R., Spaans, M., et al. 2015b, A\&A, 583, A107

Pérez-Beaupuits, J. P., Spaans, M., Hogerheijde, M. R., et al. 2010, A\&A, 510, A87

Pérez-Beaupuits, J. P., Stutzki, J., Ossenkopf, V., et al. 2015c, A\&A, 575, A9

Povich, M. S., Churchwell, E., Bieging, J. H., et al. 2009, ApJ, 696, 1278

Rauw, G., Nazé, Y., Gosset, E., et al. 2002, A\&A, 395, 499

Rauw, G., Sana, H., Spano, M., et al. 2012, A\&A, 542, A95

Risacher, C., Güsten, R., Stutzki, J., et al. 2016, A\&A, 595, A34

Sanchez-Bermudez, J., Alberdi, A., Schödel, R., et al. 2014, A\&A, 572, L1

Schöier, F. L., van der Tak, F. F. S., van Dishoeck, E. F., \& Black, J. H. 2005 A\&A, 432, 369

Schuller, F., Menten, K. M., Contreras, Y., et al. 2009, A\&A, 504, 415

Solomon, P. M., Downes, D., Radford, S. J. E., \& Barrett, J. W. 1997, ApJ, 478 144

Stacey, G. J., Jaffe, D. T., Geis, N., et al. 1993, ApJ, 404, 219

Stecklum, B., Henning, T., Eckart, A., Howell, R. R., \& Hoare, M. G. 1995, ApJ, 445, L153

Tauber, J. A., Tielens, A. G. G. M., Meixner, M., \& Goldsmith, P. F. 1994, ApJ, 422, 136

Tielens, A. G. G. M. 2010, The Physics and Chemistry of the Interstellar Medium (Cambridge, UK: Cambridge University Press)

Tielens, A. G. G. M., \& Hollenbach, D. 1985a, ApJ, 291, 747

Tielens, A. G. G. M., \& Hollenbach, D. 1985b, ApJ, 291, 722

Tothill, N. F. H., White, G. J., Matthews, H. E., et al. 2002, ApJ, 580, 285

Tothill, N. F. H., Gagné, M., Stecklum, B., \& Kenworthy, M. A. 2008, in Handbook of Star Forming Regions, Volume II: The Southern Sky, ed. B. Reipurth, 533

Urquhart, J. S., König, C., Giannetti, A., et al. 2018, MNRAS, 473, 1059

van der Tak, F. F. S., Black, J. H., Schöier, F. L., Jansen, D. J., \& van Dishoeck, E. F. 2007, A\&A, 468, 627

Walmsley, C. M., Natta, A., Oliva, E., \& Testi, L. 2000, A\&A, 364, 301

White, G. J., Nisini, B., Correia, J. C., et al. 1998, in Star Formation with the Infrared Space Observatory, eds. J. Yun, \& L. Liseau, ASP Conf. Ser., 132, 113

White, G. J., Tothill, N. F. H., Matthews, H. E., et al. 1997, A\&A, 323, 529

Woodward, C. E., Pipher, J. L., Helfer, H. L., et al. 1986, AJ, 91, 870

Woolf, N. J. 1961, PASP, 73, 206

York, D. G., Dahlstrom, J., Welty, D. E., et al. 2014, in The Diffuse Interstellar Bands, eds. J. Cami, \& N. L. J. Cox, IAU Symp., 297, 89

Young, E. T., Becklin, E. E., Marcum, P. M., et al. 2012, ApJ, 749, L17

\section{Appendix A: [C $\mathrm{CI}]$ column density}

For optically thin, thermalized [C II] emission and neglecting the effects from the background, the observed antenna temperature is found to be:

$T_{\mathrm{A}}^{*}=3.43 \times 10^{-16}\left[1+0.5 e^{91.25 / T_{\mathrm{kin}}}\right]^{-1} \frac{N([\mathrm{C} \mathrm{II}])}{\delta v} \mathrm{~K}$,

where $T_{\text {kin }}$ is the kinetic temperature, $N([\mathrm{C}$ II] $)$ is [C $\mathrm{II}]$ column density in $\mathrm{cm}^{-2}$ and $\delta v$ is the line width in $\mathrm{km} \mathrm{s}^{-1}$. 